\title{
Plant-Based Foods and Their Bioactive Compounds on Fatty Liver Disease: Effects, Mechanisms, and Clinical Application
}

\author{
Hang-Yu Li $\mathbb{D}^{1},{ }^{1}$ Ren-You Gan $\mathbb{D}^{2}{ }^{2}$ Ao Shang $\mathbb{D}^{1},{ }^{1}$ Qian-Qian Mao $\mathbb{D}^{1},{ }^{1}$ Quan-Cai Sun, ${ }^{3}$ \\ Ding-Tao Wu, ${ }^{4}$ Fang Geng, ${ }^{5}$ Xiao-Qin $\mathrm{He}^{2}$ and Hua-Bin Li ${ }^{1}{ }^{1}$ \\ ${ }^{1}$ Guangdong Provincial Key Laboratory of Food, Nutrition and Health, Department of Nutrition, School of Public Health, Sun Yat- \\ Sen University, Guangzhou 510080, China \\ ${ }^{2}$ Research Center for Plants and Human Health, Institute of Urban Agriculture, Chinese Academy of Agricultural Sciences, \\ Chengdu 610213, China \\ ${ }^{3}$ School of Food and Biological Engineering, Jiangsu University, Zhenjiang 212001, China \\ ${ }^{4}$ Institute of Food Processing and Safety, College of Food Science, Sichuan Agricultural University, Ya'an, China \\ ${ }^{5}$ Key Laboratory of Coarse Cereal Processing (Ministry of Agriculture and Rural Affairs), School of Food and Biological Engineering, \\ Chengdu University, Chengdu, China
}

Correspondence should be addressed to Ren-You Gan; ganrenyou@yahoo.com and Hua-Bin Li; lihuabin@mail.sysu.edu.cn

Received 1 December 2020; Revised 4 January 2021; Accepted 19 February 2021; Published 1 March 2021

Academic Editor: Guodong Zhang

Copyright (c) 2021 Hang-Yu Li et al. This is an open access article distributed under the Creative Commons Attribution License, which permits unrestricted use, distribution, and reproduction in any medium, provided the original work is properly cited.

\begin{abstract}
Fatty liver disease (FLD), including nonalcoholic fatty liver disease (NAFLD) and alcoholic fatty liver disease (AFLD), is a serious chronic metabolic disease that affects a wide range of people. Lipid accumulation accompanied by oxidative stress and inflammation in the liver is the most important pathogenesis of FLD. The plant-based, high-fiber, and low-fat diet has been recommended to manage FLD for a long time. This review discusses the current state of the art into the effects, mechanisms, and clinical application of plant-based foods in NAFLD and AFLD, with highlighting related molecular mechanisms. Epidemiological evidence revealed that the consumption of several plant-based foods was beneficial to alleviating FLD. Further experimental studies found out that fruits, spices, teas, coffee, and other plants, as well as their bioactive compounds, such as resveratrol, anthocyanin, curcumin, and tea polyphenols, could alleviate FLD by ameliorating hepatic steatosis, oxidative stress, inflammation, gut dysbiosis, and apoptosis, as well as regulating autophagy and ethanol metabolism. More importantly, clinical trials confirmed the beneficial effects of plant-based foods on patients with fatty liver. However, several issues need to be further studied especially the safety and effective doses of plant-based foods and their bioactive compounds. Overall, certain plant-based foods are promising natural sources of bioactive compounds to prevent and alleviate fatty liver disease.
\end{abstract}

\section{Introduction}

Fatty liver disease (FLD) has become one of the most common chronic diseases in the world, and there are two major types of FLD, including nonalcoholic fatty liver disease (NAFLD) and alcoholic fatty liver disease (AFLD) [1]. NAFLD affects approximately 1.7 billion individuals worldwide, which is almost equivalent to $25 \%$ of the global population $[2,3]$. In China, the prevalence of NAFLD has been dramatically increased from $18 \%$ to $29 \%$ in recent decades [4]. Similarly, the prevalence of NAFLD has increased by $11 \%$ in men and 3\% in women in Korea over the past 19 years
[5]. On the other hand, although the abstinence of alcohol intake is the most effective way to prevent AFLD, the truth is, from 2001 to 2016, the total prevalence of alcoholinduced fatty liver increased from $4.3 \%$ to $4.7 \%$ in the USA [6]. Moreover, a cross-sectional study reported that the prevalence of AFLD in male drinkers has increased from $22.3 \%$ to $36.6 \%$ in Japan over the last two decades [7]. In short, FLD is emerging as the priority of global health issues and intimately associated with an unhealthy lifestyle.

Both NAFLD and AFLD share the same fundamentally pathological characteristic, which is lipid accumulation accompanied by oxidative stress and inflammation in the 
liver, but the inducers are different [8]. NAFLD is commonly induced by unhealthy dietary patterns, such as a high-fat diet, over intake of fructose, and animal protein [9]. On the other hand, chronic excessive consumption of alcohol is the major trigger of AFLD [10]. Steatosis in hepatocytes was closely associated with oxidative stress, inflammation, gut dysbiosis, autophagy, and apoptosis [11-13]. All these pathological mechanisms were involved in the progression of hepatic steatosis to fibrosis, cirrhosis, and even liver cancer [14]. Further studies revealed that the disorders of several important signaling pathways played a vital role in the progression of FLD, such as adenosine $5^{\prime}$-monophosphate-activated protein kinase (AMPK), sirtuin 1 (SIRT1), peroxisome proliferator-activated receptor (PPAR), nuclear factor erythroid 2-related factor 2 (Nrf2), toll-like receptor 4 (TLR4), and nuclear factor kappa-B (NF- $\kappa \mathrm{B})$ [15-17].

The sale and consumption of plant-based food have been increased worldwide during the past two decades [18-20]. Currently, the world's market of food industry focused on how to provide more high-quality plant-based convenience foods [21]. A shift of the dietary pattern toward more plant-based food consumption is beneficial to public health [22]. Some epidemiological studies revealed that the plantbased, high-fiber, and low-fat diet was positively related to the alleviation of FLD [23-26]. Further experimental studies demonstrated that plant-based foods (such as fruits, spices, teas, and coffee) and their bioactive compounds possessed excellent properties of anti-steatosis, anti-oxidative stress, anti-inflammation, and anti-gut dysbiosis, which were related to the alleviation of FLD $[27,28]$. More importantly, researchers conducted some well-designed clinical trials to better demonstrate the biological effects of plant-based food consumption in patients with FLD $[29,30]$. Since the importance of such plants and bioactive compounds in preventing the prevalence of FLD, the safety assessment and the application issue in patients are worth discussing $[31,32]$.

To provide an updated understanding of the biological effects of plant-based foods on fatty liver diseases, this review summarized and discussed recent results from epidemiological studies, experimental studies, and clinical trials, with the emphasis focusing on their molecular mechanisms and safety assessment.

\section{Epidemiological Evidence on the Relation Between Plant-Based Foods and FLD}

Several epidemiological investigations indicated that several plant-based foods were beneficial to FLD. During 4.2 years of follow-up, a recent prospective cohort study based on 2130 NAFLD patients showed that higher consumption of fruits and vegetables decreased the risk of NAFLD in women (relative risk (RR): 0.74; 95\% confidence interval (CI): 0.59, 0.93 ) and in men (RR: 0.75 ; 95\%CI: $0.62,0.92$ ) [33]. A prospective study on 2,687 subjects showed that the higher serum level of lycopene was favorably associated with NAFLD improvement in middle-aged and elderly adults [34]. Likely, a community-based cross-sectional study involving 2,935 participants aged 40-75 years reported that lycopene was inversely relevant to NAFLD prevalence (odds ratio (OR): $0.54 ; 95 \% \mathrm{CI}: 0.42,0.68$ ) [35]. Similarly, a casecontrol study with 196 NAFLD patients and 803 controls reported that intake of spices, like garlic and onion, could decrease the risk of NAFLD (OR: 0.36; 95\%CI: 0.22, 0.56) [36]. Moreover, the OR value dropped from 1.00 to 0.71 with the increased frequency of garlic intake, suggesting that the frequency of garlic intake was inversely associated with the risk of NAFLD [37]. Besides, a multiethnic cohort study reported that the increase in coffee consumption could reduce the risks of FLD [38]. Similarly, the data from a study involving 91,436 subjects with the mean follow-up period of 2.8 years showed that the increase in coffee consumption was associated with a lower risk of FLD in Korean male [39]. In addition, several retrospectively cross-sectional studies assessed coffee intake among NAFLD patients and the result showed that the consumption of more than two cups of coffee per day could lower liver stiffness [40,41]. Furthermore, a cross-sectional study based on 58 patients showed that the dietary pattern containing other plant-based foods like whole grains and legumes could also decrease the risk of NAFLD (OR: 0.35 ; 95\%CI: 0.13, 0.93) [42]. Other cross-sectional studies with 1639 participants reported that the intake of whole grains could lower the risk of NAFLD (OR: 0.72; 95\%CI: 0.61, 0.98) [43]. Besides, a larger cross-sectional study based on 23,529 participants showed that the consumption of insoluble dietary fiber (a type of nonstarch polysaccharide widely found in whole grains) was inversely associated with the risk of NAFLD (OR: 0.7; 95\%CI: 0.61, 0.85) [44].

However, controversial results still existed among different studies. A cross-sectional study of 977 men and 1467 women from Japan showed that the association between the intake of fruit and vegetable and NAFLD was disappeared after adjustment for the body mass index [45]. What is worse, a retrospective study based on 27,214 adults showed that participants who consumed oranges over 7 times a week had a higher risk of NAFLD (OR: 1.17; 95\%CI: 1.03, 1.33) [46]. This could be due to the over intake of fructose [47]. Moreover, a cross-sectional study showed that there was no statistically significant result between coffee consumption and the risk of NAFLD progression in obese individuals [48]. Also, another cross-sectional study with 2,819 middle-aged participants showed that drinking coffee was not associated with the risk of NAFLD (OR: 0.93; 95\%CI: 0.72, 1.20) or AFLD (OR: 1.20; 95\%CI: 0.66, 2.0) [49]. Furthermore, a cohort study examining 637 patients reported that coffee consumption was not associated with the subclinical cardiovascular diseases in NAFLD patients (hazard ratio (HR): 1.05; 95\%CI: 0.91, 1.21) [50]. Additionally, the data from a two-sample Mendelian randomization analysis did not support a causal relationship between coffee intake and NAFLD risk [51].

In short, according to the results from epidemiological studies, several plant-based foods (such as garlic, onion, and whole grains), as well as their bioactive component (like insoluble dietary fiber), were beneficial to decreasing the risk of fatty liver, while excessive intake of plant-based foods containing high content of fructose (like oranges) might increase 
the risk of FLD. Besides, the relationship between coffee consumption and FLD needs further investigation.

\section{Beneficial Bioactive Compounds from Plant- Based Foods in Relation to FLD}

Increasing the consumption of more plant-based foods is an effective solution to tackle the severe burden of FLD. Recently, several bioactive compounds and phytochemicals (Figure 1) from plant-based foods are proven to make a significant influence on FLD, which are summarized below.

\subsection{Beneficial Bioactive Compounds from Fruits in Relation} to FLD. Resveratrol (3,5,4' -trihydroxy-trans-stilbene) is a natural phenolic stilbene widely found in grapes and possesses many beneficial properties [52]. It was formed via the condensation reaction of malonyl-CoA and 4-coumaroylCoA under the catalysis through the resveratrol synthase [53]. As a natural activator of SIRT1, resveratrol has been reported to prevent hepatic steatosis and even ameliorate fibrosis, and it could also improve ethanol-induced insulin resistance and regulate ethanol metabolism enzymes [5456]. Anthocyanins are a group of water-soluble flavonoids and composed of two aromatic rings linked by three carbons in an oxygenated heterocycle, with biological effects on improving insulin resistance, liver injury, and clinical progression of NAFLD [57]. Lycopene extracted from tomato is a liposoluble carotenoid without provitamin A activity, and it could improve the status of oxidative stress and inflammation and regulate metabolic processes and liver function [58].

3.2. Beneficial Bioactive Compounds from Spices in Relation to $F L D$. Curcumin ((1E,6E)-1,7-bis(4-hydroxy-3-methoxyphenyl)-1,6-heptadiene-3,5-dione) is a natural polyphenolic compound and the major curcuminoid extracted from turmeric, consisting of two aromatic O-methoxy phenolic groups, a $\beta$-dicarbonyl moiety, and a seven-carbon linker containing two enone moieties [59]. Curcumin processed a variety of bioactivities such as antioxidant, anti-inflammatory, and hepatoprotective effects, and a systematic review and dose-response meta-analysis of randomized controlled trials demonstrated that curcumin could ameliorate visceral fat and abdominal obesity in NAFLD patients [60]. Allicin (diallyl thiosulfonate), a bioactive component from the aqueous extracts of garlic, could decrease the abundance of gut microbiota, like Christensenellaceae and Ruminococcaceae, in alcoholic hepatic steatosis mice, resulting in a reduction of hepatic triacylglycerol level $[61,62]$.

\subsection{Beneficial Bioactive Compounds from Teas in Relation to} $F L D$. Tea polyphenols are the bioactive compounds extracted from tea and primarily include phenolic compounds like catechins [63]. Catechins possessed several biological effects like the improvement of oxidative stress, inflammation, diabetes, and obesity, and catechins also regulated the function of metabolic tissues like the adipose tissue and liver [64]. Epigallocatechin-3-gallate (EGCG) is the most abundant catechin in teas, and it could effectively regulate energy metabo- lism, lipid oxidation, and insulin resistance $[65,66]$. Thus, tea polyphenols could be considered a potential adjuvant therapy to modulate metabolic syndromes in FLD patients [67, 68].

3.4. Beneficial Bioactive Compounds from Coffee in Relation to FLD. Coffee is a popular beverage that contains a lot of bioactive compounds, such as caffeine, chlorogenic acid, cafestol, and kahweol [69]. Some bioactive compounds, like caffeic acid and trigonelline, are beneficial to protecting liver function due to their properties of improving liver triglyceride (TC) metabolism, oxidative stress, and fibrotic status; thus, these compounds are closely relevant to FLD [70].

3.5. Beneficial Bioactive Compounds from Other Plants in Relation to FLD. Several other plants, for example, Hongjingtian (Rhodiola rosea), milk thistle (Silybum marianum), and ginseng (Panax ginseng) are widely used functional foods and traditional herbs against FLD $[71,72]$. Salidroside (2(4-hydroxyphenyl)-ethyl- $\beta$-D-glucopyranoside) is a natural phenolic secondary metabolite from Hongjingtian (Rhodiola rosea), and it could improve lipid metabolism and antioxidative activity $[73,74]$. Silymarin is a lipophilic active complex extracted from milk thistle (Silybum marianum), and it contains several flavonolignans (like silybin, silydianin, silychristin, and isosilybin), among which, silybin had the most significant hepatoprotective effects [75, 76]. Ginsenosides are amorphous compounds extracted from ginseng (Panax ginseng). There are two major groups of ginsenosides, the protopanaxadiol and protopanaxatriol groups, and they could suppress appetite by affecting the ventromedial hypothalamic nucleus, while inhibiting the absorption of fat in the gut $[77,78]$.

\section{Mechanisms of Plant-Based Foods and Their Bioactive Compounds on FLD}

The effects of plant-based foods (such as fruits, spices, teas, coffee, and other plants) and their bioactive compounds (such as resveratrol, anthocyanins, lycopene, curcumin, and tea polyphenols) on FLD have been widely investigated by in vitro and in vivo experimental studies. The potential molecular mechanisms are discussed and highlighted below.

\subsection{Fruits and FLD}

4.1.1. Mechanisms of Fruits and Their Bioactive Compounds on NAFLD. Bioactive food constituents were recognized as new treatment approaches in the modulation of NAFLD. Resveratrol is widely found in plants like grapes and could attenuate NAFLD by inhibiting lipogenesis and oxidative stress in HepG2 cells [79]. Meanwhile, resveratrol is also a main compound in red wine and could improve the hepatic steatosis and redox homeostasis in HepG2 cells by upregulating the protein kinase A- (PKA-) AMPK-PPAR- $\alpha$ signaling pathway and regulating the epigenetic modification of the Nrf2 signaling pathway $[80,81]$. Similarly, rutin from tomato could inhibit oxidative stress and autophagy in the NAFLD cell model by upregulating the PPAR- $\alpha$ signaling pathway, accompanied by reducing the level of TG [82]. Besides, the 


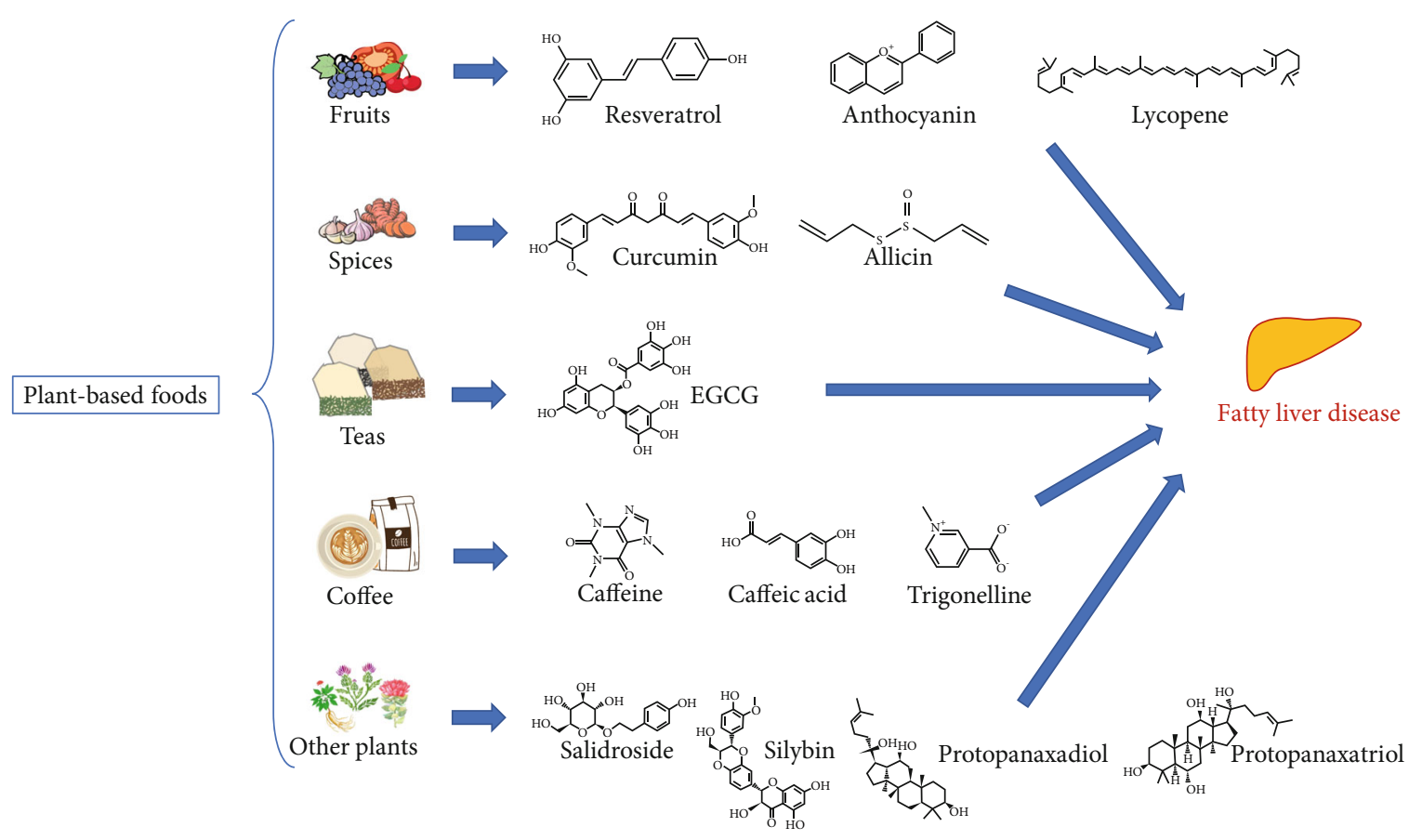

FIGURE 1: Bioactive compounds from plant-based foods relevant to FLD.

administration of anthocyanin extracted from sweet cherry could alleviate NAFLD in HepG2 cells and LO2 liver cells through inducing autophagy by downregulating phosphorylation of the mammalian target of rapamycin (mTOR) and protein kinase $\mathrm{B}(\mathrm{Akt})$, while upregulating the phosphorylation of AMPK [83].

In addition, in vivo studies also showed that the oral administration of resveratrol and anthocyanin extracted from grape improved lipid metabolism, oxidative stress, and inflammation in the NAFLD mouse model by downregulating PPAR- $\gamma$ while upregulating Nrf2 and SIRT1 signaling pathways, accompanied by decreasing the expression of fatty acid synthase (FAS) and sterol regulatory element-binding transcription factor 1c (SREBP-1c) $[81,84]$. Besides, the consumption of tomato juice could alleviate NAFLD in Sprague-Dawley (SD) rats by increasing the abundance of Lactobacillus and diminishing the ratio of acetate to propionate [85]. Furthermore, lycopene extracted from tomato could dose-dependently improve the liver function and lipid profiles in the NAFLD rat model by decreasing the levels of cytochrome P450 2E1 (CYP2E1), malondialdehyde (MDA), and tumor necrosis factor- $\alpha$ (TNF- $\alpha)$ in the liver [86-88]. Other fruits, like mulberry, Açai, and acerola cherry, also suppressed hepatic steatosis, oxidative stress, and inflammation in animal experiments [89-91].

In summary, several fruits (like grape, cherry, tomato, and mulberry) and their bioactive compounds (like resveratrol, anthocyanin, and lycopene) could be promising agents against NAFLD due to their outstanding effects on regulating lipid metabolism, oxidative stress, inflammation, and gut microbiota (Table 1), with regulating AMPK, PPAR $-\alpha / \gamma$, Nrf2, mTOR, and Akt signaling pathways (Figure 2).
4.1.2. Mechanisms of Fruits and Their Bioactive Compounds on AFLD. Several fruits and their bioactive compounds show protective effects on AFLD in vitro. The administration of resveratrol from fruits could alleviate AFLD in HepG2 cells by activating the AMPK-lipin1 signaling pathway, accompanied by the decreased expression of SREBP-1c and lipin 1 [92]. Similarly, some natural flavonoids (like myricetin and myricitrin) extracted from berries could alleviate alcoholinduced lipid accumulation, oxidative stress, and inflammation in AML12 cells by enhancing the phosphorylation of AMPK and reducing the expression of FAS and SREBP-1c $[93,94]$.

Fruit and their bioactive compounds also show protective effects on AFLD in vivo. The consumption of lemon juice could dose-dependently alleviate alcohol-induced lipid accumulation and peroxidation in the liver [95]. Also, oral intake of blueberry juice could alleviate AFLD in C57BL/6J mice through inhibiting apoptosis by upregulating SIRT1 and downregulating the forkhead box protein O1 (FOXO1) signaling pathway [96]. Moreover, polyphenols extracted from blueberry could accelerate lipid clearance to ameliorate hepatic steatosis in C57BL/6J mice by promoting autophagy [97]. Furthermore, phenolic extracts from lychee could alleviate AFLD in C57BL/6 mice by improving hepatic steatosis, oxidative stress, and gut dysbiosis, with upregulating the Nrf2 pathway and decreasing the levels of cytochrome c, caspase-3, and the ratio of $\mathrm{Bcl} 2$-associated $\mathrm{X}$ protein (Bax) to $\mathrm{B}$ cell lymphoma-2 (Bcl-2) $[98,99]$. Additionally, the aqueous extract of mulberry could ameliorate AFLD in SD rats by accelerating ethanol degradation and decreasing the ratio of Firmicutes to Bacteroidetes in the gut [100]. Other fruits like Açai could alleviate alcohol-induced liver injury in Wistar rats by suppressing oxidative stress and inflammatory 
TABLE 1: The protective effects and molecular mechanisms of plant-based foods against NAFLD and AFLD in experimental studies.

\begin{tabular}{|c|c|c|c|c|c|c|c|}
\hline $\begin{array}{l}\text { Plant-based } \\
\text { foods }\end{array}$ & $\begin{array}{l}\text { Bioactive } \\
\text { component }\end{array}$ & $\begin{array}{l}\text { Study } \\
\text { type }\end{array}$ & Models & Doses & Main effects & Molecular mechanisms & Ref \\
\hline \multicolumn{8}{|c|}{ Fruits-NAFLD } \\
\hline Grape & Resveratrol & $\begin{array}{c}\text { In } \\
\text { vitro }\end{array}$ & HepG2 cells & $\begin{array}{l}12.5,25,50 \\
100 \mu \mathrm{M}\end{array}$ & $\begin{array}{l}\text { Inhibiting lipogenesis } \\
\text { and proliferation }\end{array}$ & Not mentioned & {$[79]$} \\
\hline \multirow[t]{3}{*}{$\begin{array}{l}\text { Grape (red } \\
\text { wine) }\end{array}$} & Resveratrol & $\begin{array}{l}\text { In } \\
\text { vitro, } \\
\text { in vivo }\end{array}$ & $\begin{array}{l}\text { HepG2 cells } \\
\text { SD rats }\end{array}$ & $\begin{array}{c}40 \mu \mathrm{M} \\
100 \mathrm{mg} / \mathrm{kg}\end{array}$ & $\begin{array}{l}\text { Alleviating lipid } \\
\text { accumulation and } \\
\text { oxidative stress }\end{array}$ & $\begin{array}{l}\text { Activating the PKA-AMPK- } \\
\text { PPAR- } \alpha \text { pathway }\end{array}$ & {$[80]$} \\
\hline & Resveratrol & $\begin{array}{l}\text { In } \\
\text { vitro, } \\
\text { in vivo }\end{array}$ & $\begin{array}{l}\text { HepG2 cells, } \\
\text { C57BL/6 } \\
\text { mice }\end{array}$ & $\begin{array}{l}20 \mu \mathrm{M} \text { for cells } \\
0.4 \% \text { in the } \\
\text { chow }\end{array}$ & $\begin{array}{l}\text { Reducing the expression } \\
\text { of FAS and SREBP-1c } \\
\text { genes }\end{array}$ & $\begin{array}{l}\text { Inhibiting methylation of } \mathrm{Nrf} 2 \\
\text { promoter }\end{array}$ & {$[81]$} \\
\hline & Rutin & $\begin{array}{l}\text { In } \\
\text { vitro, } \\
\text { in vivo }\end{array}$ & $\begin{array}{l}\text { HepG2 cells, } \\
\text { RAW } 246.7 \\
\text { cells, } \\
\text { C57BL/6 } \\
\text { mice }\end{array}$ & $\begin{array}{l}10,20,40 \mu \mathrm{M} \\
200 \mathrm{mg} / \mathrm{kg}\end{array}$ & $\begin{array}{c}\text { Inhibiting lipogenesis, } \\
\text { oxidative injuries, and } \\
\text { autophagy }\end{array}$ & $\begin{array}{l}\text { Activating the PPAR- } \alpha \\
\text { pathway }\end{array}$ & {$[82]$} \\
\hline Sweet cherry & Anthocyanins & $\begin{array}{l}\text { In } \\
\text { vitro }\end{array}$ & $\begin{array}{l}\text { HepG2 cells } \\
\text { LO2 cells }\end{array}$ & $\begin{array}{c}100,200 \\
300 \mu \mathrm{g} / \mathrm{mL}\end{array}$ & Inducing autophagy & $\begin{array}{c}\text { Activating AMPK and } \\
\text { inhibiting mTOR and Akt } \\
\text { pathways }\end{array}$ & {$[83]$} \\
\hline Grape & $\begin{array}{l}\text { Polymerized } \\
\text { anthocyanin }\end{array}$ & In vivo & $\begin{array}{l}\mathrm{C} 57 \mathrm{BL} / 6 \mathrm{~J} \\
\text { mice }\end{array}$ & $400 \mathrm{mg} / \mathrm{kg}$ & $\begin{array}{l}\text { Improving liver } \\
\text { function, dyslipidemia, } \\
\text { and hepatic steatosis }\end{array}$ & $\begin{array}{l}\text { Activating Nrf2 and SIRT1 and } \\
\text { inhibiting PPAR- } \gamma \text { pathways }\end{array}$ & {$[84]$} \\
\hline \multirow[t]{2}{*}{ Tomato } & & In vivo & SD rats & Freely drink & Alleviating gut dysbiosis & $\begin{array}{l}\text { Increasing Lactobacillus } \\
\text { abundance and diminishing the } \\
\text { acetate/propionate ratio }\end{array}$ & {$[85]$} \\
\hline & Lycopene & In vivo & $\begin{array}{c}\text { SD rats } \\
\text { Wistar rats }\end{array}$ & $5,10,20 \mathrm{mg} / \mathrm{kg}$ & $\begin{array}{l}\text { Alleviating liver injury, } \\
\text { lipid accumulation, fat } \\
\text { infiltration, and } \\
\text { oxidative stress }\end{array}$ & $\begin{array}{l}\text { Reverting activities of SOD, } \\
\text { GSH, and CAT and decreasing } \\
\text { TNF- } \alpha \text { and CYP2E1 levels }\end{array}$ & $\begin{array}{c}{[86-} \\
88]\end{array}$ \\
\hline $\begin{array}{l}\text { Acerola } \\
\text { cherry }\end{array}$ & Polysaccharide & In vivo & $\begin{array}{l}\mathrm{C} 57 \mathrm{BL} / 6 \\
\text { mice }\end{array}$ & $\begin{array}{c}200,400 \\
800 \mathrm{mg} / \mathrm{kg} / \text { day }\end{array}$ & $\begin{array}{l}\text { Improving } \\
\text { mitochondrial function, } \\
\text { lipogenesis, oxidative } \\
\text { stress, and inflammation }\end{array}$ & $\begin{array}{l}\text { Inhibiting SREBP-1c and } \\
\text { activating PGC- } 1 \alpha \text { and Nrf2 } \\
\text { pathways }\end{array}$ & [89] \\
\hline Mulberry & & In vivo & SD rats & $\begin{array}{c}100 \\
200 \mathrm{mg} / \mathrm{kg}\end{array}$ & $\begin{array}{l}\text { Alleviating liver } \\
\text { damage, dyslipidemia, } \\
\text { and oxidative stress }\end{array}$ & $\begin{array}{l}\text { Improving mitochondrial } \\
\text { function }\end{array}$ & {$[90]$} \\
\hline Açai & & In vivo & Fischer rats & $20 \mathrm{~g} / \mathrm{kg}$ & $\begin{array}{l}\text { Alleviating steatosis and } \\
\text { inflammation }\end{array}$ & $\begin{array}{l}\text { Reducing liver enzymes and } \\
\text { increasing GSH/GSSG }\end{array}$ & [91] \\
\hline \multicolumn{8}{|l|}{ Fruits-AFLD } \\
\hline & Resveratrol & $\begin{array}{l}\text { In } \\
\text { vitro }\end{array}$ & HepG2 cell & $\begin{array}{l}5,15,45 \\
135 \mu \mathrm{M}\end{array}$ & $\begin{array}{l}\text { Reducing lipid } \\
\text { accumulation }\end{array}$ & $\begin{array}{l}\text { Activating the AMPK-lipin1 } \\
\text { pathway }\end{array}$ & {$[92]$} \\
\hline & $\begin{array}{l}\text { Myricitrin } \\
\text { Myricetin }\end{array}$ & $\begin{array}{c}\text { In } \\
\text { vitro }\end{array}$ & AML12 cells & $\begin{array}{l}5,10,20 \\
40 \mu \mathrm{M} \\
60 \mu \mathrm{M}\end{array}$ & $\begin{array}{l}\text { Alleviating steatosis, } \\
\text { oxidative stress, and } \\
\text { inflammation }\end{array}$ & Activating the AMPK pathway & $\begin{array}{l}{[93,} \\
94]\end{array}$ \\
\hline Lemon & & In vivo & $\begin{array}{l}\mathrm{C} 57 \mathrm{BL} / 6 \\
\text { mice }\end{array}$ & $10 \mathrm{~mL} / \mathrm{kg}$ & $\begin{array}{l}\text { Alleviating lipid } \\
\text { accumulation and lipid } \\
\text { peroxidation }\end{array}$ & $\begin{array}{l}\text { Decreasing the levels of SOD } \\
\text { and CAT }\end{array}$ & [95] \\
\hline Blueberry & Polyphenols & In vivo & $\begin{array}{l}\text { C57BL/6J } \\
\text { mice }\end{array}$ & $\begin{array}{c}1.5 \mathrm{~mL} / 100 \mathrm{~g} \\
100, \\
200 \mathrm{mg} / \mathrm{kg} / \text { day }\end{array}$ & $\begin{array}{l}\text { Inhibiting apoptosis and } \\
\text { promoting autophagy }\end{array}$ & $\begin{array}{l}\text { Activating SIRT1 and } \\
\text { inhibiting FOXO1 pathways }\end{array}$ & $\begin{array}{l}{[96,} \\
97]\end{array}$ \\
\hline Lychee & Phenols & In vivo & $\begin{array}{l}\mathrm{C} 57 \mathrm{BL} / 6 \\
\text { mice }\end{array}$ & $\begin{array}{c}150, \\
300 \mathrm{mg} / \mathrm{kg}\end{array}$ & $\begin{array}{l}\text { Alleviating steatosis, } \\
\text { oxidative stress, and gut } \\
\text { dysbiosis }\end{array}$ & $\begin{array}{c}\text { Activating the Nrf2 pathway, } \\
\text { decreasing cytochrome c, } \\
\text { caspase- } 3 \text { activities, and } \\
\text { Bax/Bcl-2 ratio }\end{array}$ & $\begin{array}{l}{[98,} \\
99]\end{array}$ \\
\hline Mulberry & & In vivo & $\mathrm{SD}$ rats & $0.3 \mathrm{~g} / \mathrm{kg}$ & & & [100] \\
\hline
\end{tabular}


Table 1: Continued.

\begin{tabular}{|c|c|c|c|c|c|c|c|}
\hline $\begin{array}{l}\text { Plant-based } \\
\text { foods }\end{array}$ & $\begin{array}{l}\text { Bioactive } \\
\text { component }\end{array}$ & $\begin{array}{l}\text { Study } \\
\text { type }\end{array}$ & Models & Doses & Main effects & Molecular mechanisms & Ref \\
\hline \multirow{6}{*}{$\begin{array}{l}\text { Açai } \\
\text { Spices-NAFLI }\end{array}$} & & & & & $\begin{array}{l}\text { Improving steatosis, gut } \\
\text { dysbiosis, and glucose } \\
\text { metabolism }\end{array}$ & $\begin{array}{l}\text { Accelerating ethanol } \\
\text { degradation, decreasing ratio of } \\
\text { Firmicutes to Bacteroidetes }\end{array}$ & \\
\hline & & In vivo & Wistar rats & $1 \mathrm{~mL} / 100 \mathrm{~g}$ & $\begin{array}{l}\text { Alleviating oxidative } \\
\text { stress and inflammation }\end{array}$ & Inhibiting the NF- $\kappa \mathrm{B}$ pathway & [101] \\
\hline & & & & & & & \\
\hline & Curcumin & $\begin{array}{c}\text { In } \\
\text { vitro } \\
\text { In vivo }\end{array}$ & $\begin{array}{l}\text { Primary liver } \\
\text { cells } \\
\text { C57BL/6 } \\
\text { mice }\end{array}$ & $\begin{array}{c}10 \mu \mathrm{M} \\
50,100 \mathrm{mg} / \mathrm{kg}\end{array}$ & $\begin{array}{l}\text { Regulating bile acids } \\
\text { and exogenous } \\
\text { xenobiotic metabolism }\end{array}$ & $\begin{array}{l}\text { Increasing Nrf } 2 \text { and FXR and } \\
\text { decreasing LXR } \alpha \text { levels }\end{array}$ & [102] \\
\hline & Curcumin & $\begin{array}{l}\text { In } \\
\text { vitro } \\
\text { In vivo }\end{array}$ & $\begin{array}{l}\mathrm{PBM} \text { cells } \\
\mathrm{C} 57 \mathrm{BL} / 6 \mathrm{~J} \\
\text { mice }\end{array}$ & $\begin{array}{l}30 \mu \mathrm{M} \text { for cells } \\
2 \mathrm{~g} / \mathrm{kg} \text { in the } \\
\text { chow }\end{array}$ & $\begin{array}{l}\text { Preventing intrahepatic } \\
\text { CD4+ cell } \\
\text { accumulation, oxidative } \\
\text { stress, and inflammation }\end{array}$ & $\begin{array}{l}\text { Inhibiting the production of } \\
\text { ROS, TNF- } \alpha \text {, and IFN- } \gamma\end{array}$ & [103] \\
\hline & Curcumin & $\begin{array}{c}\text { In } \\
\text { vitro } \\
\text { In vivo }\end{array}$ & $\begin{array}{l}\text { AML12 cells } \\
\text { C57BL/6J } \\
\text { mice }\end{array}$ & $\begin{array}{c}0.3,3 \mu \mathrm{M} \\
100 \mathrm{mg} / \mathrm{kg}\end{array}$ & $\begin{array}{c}\text { Alleviating lipid } \\
\text { accumulation, oxidative } \\
\text { stress, and inflammation }\end{array}$ & $\begin{array}{l}\text { Inhibiting O-GlcNAcylation of } \\
\text { NF- } \kappa \text { B and upregulating the } \\
\text { SIRT1-AMPK-ACC pathway }\end{array}$ & {$[104]$} \\
\hline Onion & & In vivo & $\mathrm{SD}$ rats & $7 \% w / w$ & $\begin{array}{l}\text { Alleviating steatosis, } \\
\text { ballooning, and lobular } \\
\text { and portal inflammation }\end{array}$ & $\begin{array}{l}\text { Decreasing levels of TNF- } \alpha \text {, } \\
\text { ALT, AST, TG, insulin, and } \\
\text { glucose }\end{array}$ & $\begin{array}{l}{[105,} \\
106]\end{array}$ \\
\hline \multicolumn{8}{|l|}{ Spices-AFLD } \\
\hline Garlic & Allicin & In vivo & $\begin{array}{l}\text { C57BL/6 } \\
\text { mice }\end{array}$ & $\begin{array}{c}5, \\
20 \mathrm{mg} / \mathrm{kg} / \text { day }\end{array}$ & $\begin{array}{l}\text { Alleviating oxidative } \\
\text { stress and inflammation }\end{array}$ & $\begin{array}{l}\text { Reducing the levels of SERBP- } \\
\text { 1c, CYP2E1, TNF- } \alpha \text {, IL- } 1 \beta \text {, and } \\
\text { IL-6, increasing the levels of } \\
\text { GSH, CAT, and the activity of } \\
\text { ADH }\end{array}$ & [107] \\
\hline Garlic & Allicin & In vivo & $\begin{array}{l}\text { C57BL/6 } \\
\text { mice }\end{array}$ & $\begin{array}{c}5, \\
20 \mathrm{mg} / \mathrm{kg} / \text { day }\end{array}$ & $\begin{array}{l}\text { Alleviating steatosis, } \\
\text { inflammation, and gut } \\
\text { dysbiosis }\end{array}$ & $\begin{array}{l}\text { Inhibiting the LPS-TLR4 } \\
\text { pathway }\end{array}$ & {$[62]$} \\
\hline \multirow[t]{2}{*}{ Ginger } & & In vivo & Wistar rats & $50 \mathrm{mg} / \mathrm{kg}$ & $\begin{array}{l}\text { Alleviating lipid } \\
\text { accumulation and liver } \\
\text { enzyme changes }\end{array}$ & $\begin{array}{l}\text { Increasing the expression of } \\
\text { HNF4A and decreasing the } \\
\text { expression of the PTP1B gene }\end{array}$ & [108] \\
\hline & Curcumin & In vivo & $\begin{array}{l}\text { Kunming } \\
\text { mice }\end{array}$ & $60 \mathrm{mg} / \mathrm{kg}$ & $\begin{array}{l}\text { Suppressing fatty acid } \\
\text { biosynthesis and } \\
\text { pentose glucuronate } \\
\text { pathway }\end{array}$ & $\begin{array}{l}\text { Inhibiting the metabolisms of } \\
\text { glyoxylate, dicarboxylate, and } \\
\text { pyruvate }\end{array}$ & [109] \\
\hline \multicolumn{8}{|l|}{ Tea-NAFLD } \\
\hline Green tea & Catechins & $\begin{array}{l}\text { In } \\
\text { vitro } \\
\text { In vivo }\end{array}$ & $\begin{array}{l}\text { HepG2 cells } \\
\text { C57BL/6 } \\
\text { mice }\end{array}$ & $\begin{array}{l}2 \mu \mathrm{M} \text { and } \\
0.19 \% \\
500 \mathrm{mg} / \mathrm{kg}\end{array}$ & $\begin{array}{l}\text { Alleviating lipid } \\
\text { accumulation, } \\
\text { increasing gene } \\
\text { expression related to } \\
\text { catabolism of TG and } \\
\text { fatty acid }\end{array}$ & $\begin{array}{l}\text { Downregulating miR-34a and } \\
\text { upregulating miR-194 }\end{array}$ & [110] \\
\hline $\begin{array}{l}\text { Raw bowl } \\
\text { dark tea }\end{array}$ & Polyphenol & $\begin{array}{l}\text { In } \\
\text { vitro } \\
\text { In vivo }\end{array}$ & $\begin{array}{l}\text { 3T3-L1 } \\
\text { preadipocytes } \\
\text { C57BL/6N } \\
\text { mice }\end{array}$ & $\begin{array}{c}200 \mu \mathrm{g} / \mathrm{mL} \\
50, \\
100 \mathrm{mg} / \mathrm{kg} / \text { day }\end{array}$ & $\begin{array}{l}\text { Alleviating lipid } \\
\text { accumulation, oxidative } \\
\text { stress, and inflammation } \\
\text { and improving the } \\
\text { intestinal environment }\end{array}$ & $\begin{array}{l}\text { Increasing the levels of } \\
\text { occludin, ZO-1, Bacteroides, } \\
\text { and Akkermansia and reducing } \\
\text { the level of Firmicutes }\end{array}$ & [111] \\
\hline Hao Ling tea & $\begin{array}{l}\text { Polyphenol } \\
\text { Caffeine }\end{array}$ & $\begin{array}{l}\text { In } \\
\text { vitro } \\
\text { In vivo }\end{array}$ & $\begin{array}{l}\text { Primary liver } \\
\text { cells } \\
\text { Wistar rats }\end{array}$ & $\begin{array}{l}100,250 \\
500 \mu \mathrm{g} / \mathrm{mL} \\
10 \% \text { in the } \\
\quad \text { drink }\end{array}$ & $\begin{array}{l}\text { Alleviating hepatic } \\
\text { steatosis and oxidative } \\
\text { stress }\end{array}$ & $\begin{array}{l}\text { Inhibiting the production of } \\
\text { mitochondrial ROS }\end{array}$ & {$[112]$} \\
\hline Green tea & $\begin{array}{l}\text { Phenols } \\
\text { Flavonoids }\end{array}$ & In vivo & Wistar rats & $300 \mathrm{mg} / \mathrm{kg}$ & $\begin{array}{l}\text { Improving } \\
\text { hyperlipidemia and } \\
\text { oxidative stress }\end{array}$ & Increasing the activity of SOD & [113] \\
\hline
\end{tabular}


TABle 1: Continued.

\begin{tabular}{|c|c|c|c|c|c|c|c|}
\hline $\begin{array}{l}\text { Plant-based } \\
\text { foods }\end{array}$ & $\begin{array}{l}\text { Bioactive } \\
\text { component }\end{array}$ & $\begin{array}{l}\text { Study } \\
\text { type }\end{array}$ & Models & Doses & Main effects & Molecular mechanisms & Ref \\
\hline Green tea & Polyphenol & In vivo & Zucker rats & $200 \mathrm{mg} / \mathrm{kg}$ & $\begin{array}{l}\text { Decreasing lipogenesis, } \\
\text { levels of insulin, glucose, } \\
\text { liver enzymes, TNF- } \alpha \text {, } \\
\text { and IL-6 }\end{array}$ & $\begin{array}{l}\text { Upregulating the AMPK } \\
\text { pathway }\end{array}$ & [114] \\
\hline $\begin{array}{l}\text { Ning Hong } \\
\text { black tea }\end{array}$ & & In vivo & SD rats & $\begin{array}{l}2 \% \text { in the } \\
\text { chow }\end{array}$ & $\begin{array}{l}\text { Decreasing the body fat } \\
\text { ratio and the number of } \\
\text { lipid droplets in the liver }\end{array}$ & $\begin{array}{l}\text { Upregulating expression of } \\
\text { PPAR- } \alpha \text { and MTP, promoting } \\
\text { fatty acid } \beta \text {-oxidation and } \\
\text { VLDL synthesis }\end{array}$ & [115] \\
\hline \multicolumn{8}{|l|}{ Tea-AFLD } \\
\hline Green tea & & In vivo & $\begin{array}{l}\text { Kunming } \\
\text { mice }\end{array}$ & $10 \mathrm{~mL} / \mathrm{kg}$ & $\begin{array}{l}\text { Improving ethanol } \\
\text { metabolism and liver } \\
\text { function }\end{array}$ & $\begin{array}{l}\text { Increasing the activities of } \\
\mathrm{ADH} \text { and ALDH }\end{array}$ & [116] \\
\hline Green tea & $\begin{array}{l}\text { Catechins } \\
\text { Caffeine }\end{array}$ & In vivo & Wistar rats & $20 \mathrm{~mL} / \mathrm{kg} /$ day & $\begin{array}{l}\text { Alleviating lipogenesis } \\
\text { and oxidative injury }\end{array}$ & $\begin{array}{l}\text { Reducing levels of SREBP-1c, } \\
\text { FAS, CYP2E1, and NADPH } \\
\text { oxidase p47phox protein }\end{array}$ & [117] \\
\hline Green tea & EGCG & In vivo & Wistar rats & $300 \mathrm{mg} / \mathrm{kg} /$ day & $\begin{array}{l}\text { Alleviating oxidative } \\
\text { stress and necrosis }\end{array}$ & $\begin{array}{l}\text { Decreasing levels of TNF- } \alpha \text { and } \\
\text { 4-hydroxynonenal }\end{array}$ & [118] \\
\hline Green tea & EGCG & In vivo & Wistar rats & $3 \mathrm{~g} / \mathrm{L}$ & $\begin{array}{c}\text { Improving fatty liver } \\
\text { and the levels of ALT } \\
\text { and AST }\end{array}$ & $\begin{array}{l}\text { Increasing the phosphorylation } \\
\text { of ACC and the level of CPT-1 }\end{array}$ & [119] \\
\hline Green tea & Catechin & In vivo & Wistar rats & $50 \mathrm{mg} / \mathrm{kg} /$ day & $\begin{array}{l}\text { Alleviating fatty } \\
\text { changes, liver } \\
\text { dysfunction, and } \\
\text { oxidative stress }\end{array}$ & Inhibiting the NF- $\kappa \mathrm{B}$ pathway & [120] \\
\hline \multicolumn{8}{|c|}{ Coffee-NAFLD } \\
\hline & Caffeic acid & $\begin{array}{c}\text { In } \\
\text { vitro } \\
\text { In vivo }\end{array}$ & $\begin{array}{l}\text { AML } 12 \\
\text { C57BL/6 } \\
\text { mice }\end{array}$ & $\begin{array}{c}12.5,25,50 \\
100,200 \mu \mathrm{M} \\
50 \mathrm{mg} / \mathrm{kg} / \text { day }\end{array}$ & $\begin{array}{l}\text { Alleviating steatosis } \\
\text { endoplasmic reticulum } \\
\text { stress and increasing } \\
\text { autophagy }\end{array}$ & Activating the Akt pathway & [121] \\
\hline & Trigonelline & $\begin{array}{l}\text { In } \\
\text { vitro } \\
\text { In vivo }\end{array}$ & $\begin{array}{l}\text { AML } 12 \\
\text { HepG2 cells } \\
\text { C57BL/6J } \\
\text { mice }\end{array}$ & $\begin{array}{l}50,200 \mu \mathrm{M} \\
50 \mathrm{mg} / \mathrm{kg}\end{array}$ & $\begin{array}{l}\text { Alleviating steatosis and } \\
\text { lipotoxicity and } \\
\text { promoting autophagy }\end{array}$ & $\begin{array}{c}\text { Deceasing the phosphorylation } \\
\text { of mTOR and the expression of } \\
\text { PPAR- } \gamma \text {, SREBP-1, perilipin, } \\
\text { and CD36 }\end{array}$ & [122] \\
\hline Coffee & Caffeine & $\begin{array}{c}\text { In } \\
\text { vitro } \\
\text { In vivo }\end{array}$ & $\begin{array}{l}\text { HepG2 cells } \\
\text { C57BL/6 } \\
\text { mice }\end{array}$ & $\begin{array}{c}2 \mathrm{mM} \\
10,20 \mathrm{mg} / \mathrm{kg}\end{array}$ & Alleviating steatosis & $\begin{array}{l}\text { Activating the SIRT3-AMPK- } \\
\text { ACC pathway }\end{array}$ & [123] \\
\hline Coffee & Caffeine & In vivo & $\begin{array}{l}\text { Tsumura } \\
\text { Suzuki } \\
\text { nonobese } \\
\text { mice }\end{array}$ & $\begin{array}{l}1 \mathrm{~g} / \mathrm{L} \text {, freely } \\
\text { drink }\end{array}$ & $\begin{array}{l}\text { Inhibiting pancreatic- } \beta \\
\text { cell damage and } \\
\text { nonalcoholic } \\
\text { steatohepatitis }\end{array}$ & Not mentioned & [124] \\
\hline Coffee & & In vivo & Wistar rats & $1000 \mathrm{mg} / \mathrm{kg}$ & $\begin{array}{l}\text { Alleviating steatosis, } \\
\text { insulin resistance, and } \\
\text { oxidative stress }\end{array}$ & $\begin{array}{l}\text { Upregulating the expression of } \\
\text { PPAR- } \alpha\end{array}$ & [125] \\
\hline \multirow[t]{2}{*}{ Coffee } & & In vivo & $\begin{array}{l}\text { C57BL/6J } \\
\text { mice }\end{array}$ & $\begin{array}{l}4: 1, v / w, \text { freely } \\
\text { drink }\end{array}$ & $\begin{array}{l}\text { Improving liver fat } \\
\text { oxidation, intestinal } \\
\text { cholesterol efflux, } \\
\text { energy metabolism, and } \\
\text { gut permeability }\end{array}$ & $\begin{array}{l}\text { Upregulating the expression of } \\
\text { PPAR- } \alpha \text {, acyl-CoA oxidase-1, } \\
\text { ABCA1, ABCG1, zonulin-1, } \\
\text { claudin, and peptide YY, as well } \\
\text { as increasing the abundance of } \\
\text { Alcaligenaceae }\end{array}$ & [126] \\
\hline & Trigonelline & In vivo & SD rats & $40 \mathrm{mg} / \mathrm{kg} /$ day & $\begin{array}{l}\text { Alleviating steatosis and } \\
\text { the damage degree of the } \\
\text { liver }\end{array}$ & $\begin{array}{l}\text { Increasing the level of SOD and } \\
\text { the expression of } \mathrm{Bcl}-2\end{array}$ & [127] \\
\hline Coffee & Caffeine & In vivo & $\begin{array}{l}\text { C57BL/6J } \\
\text { mice }\end{array}$ & $\begin{array}{l}0.5 \mathrm{mg} / \mathrm{mL} \text {, } \\
\text { freely drink }\end{array}$ & Alleviating steatosis & $\begin{array}{l}\text { Activating STAT3 in the liver } \\
\text { and increasing IL- } 6 \text { in } \\
\text { circulation }\end{array}$ & [128] \\
\hline
\end{tabular}


TABle 1: Continued.

\begin{tabular}{|c|c|c|c|c|c|c|c|}
\hline $\begin{array}{l}\text { Plant-based } \\
\text { foods }\end{array}$ & $\begin{array}{l}\text { Bioactive } \\
\text { component }\end{array}$ & $\begin{array}{l}\text { Study } \\
\text { type }\end{array}$ & Models & Doses & Main effects & Molecular mechanisms & Ref \\
\hline Coffee & Caffeine & In vivo & SD rats & $\begin{array}{l}8 \mathrm{~g} / 180 \mathrm{~mL} \text { in } \\
\text { drinking } \\
\text { water, } \\
0.18 \mathrm{~g} / \mathrm{kg} \text { in } \\
\text { diet }\end{array}$ & Alleviating steatosis & $\begin{array}{c}\text { Decreasing the } \\
\text { phosphorylation of mTOR and } \\
\text { increasing the level of nuclear } \\
\text { lipin1 }\end{array}$ & $\begin{array}{l}{[129,} \\
130]\end{array}$ \\
\hline Coffee & & In vivo & $\begin{array}{l}\text { Wistar rats } \\
\text { C57BL/6J } \\
\text { mice }\end{array}$ & $6 \mathrm{~g} / \mathrm{kg}$ & $\begin{array}{l}\text { Improving the gut } \\
\text { permeability and } \\
\text { intestinal barrier } \\
\text { function }\end{array}$ & $\begin{array}{l}\text { Increasing the expression of } \\
\text { occludin and ZO-1, decreasing } \\
\text { the expression of TLR } 4\end{array}$ & [131] \\
\hline Coffee & Caffeine & In vivo & Wistar rats & $\begin{array}{c}30,60 \\
120 \mathrm{mg} / \mathrm{kg} / \text { day }\end{array}$ & $\begin{array}{l}\text { Increasing the } \\
\text { susceptibility of NAFLD } \\
\text { in offspring }\end{array}$ & $\begin{array}{l}\text { Inhibiting the expression of } \\
\text { SIRT1 }\end{array}$ & [132] \\
\hline \multicolumn{8}{|c|}{ Coffee-AFLD } \\
\hline Coffee & Caffeine & In vivo & $\begin{array}{l}\text { Kunming } \\
\text { mice }\end{array}$ & $5,10,20 \mathrm{mg} / \mathrm{kg}$ & $\begin{array}{l}\text { Alleviating hepatic cell } \\
\text { damage, steatosis, and } \\
\text { inflammatory response }\end{array}$ & $\begin{array}{l}\text { Decreasing the expression of } \\
\text { SERBP-1c, Fas, ACC, SCD 1, } \\
\text { and the levels of TNF- } \alpha \text {, IL- } \\
1 \beta / 6, \text { IFN- } \gamma \text {, and MCP- } 1\end{array}$ & [133] \\
\hline Coffee & Caffeine & In vivo & $\mathrm{SD}$ rats & $\begin{array}{c}5,10, \text { and } \\
20 \mathrm{mg} / \mathrm{kg} / \text { day }\end{array}$ & $\begin{array}{l}\text { Inhibiting the activation } \\
\text { of hepatic stellate cell }\end{array}$ & Inhibiting the PKA pathway & [134] \\
\hline Coffee & Caffeic acid & In vivo & Wistar rats & $12 \mathrm{mg} / \mathrm{kg} /$ day & $\begin{array}{l}\text { Decreasing the levels of } \\
\text { TG, TC, free fatty acids, } \\
\text { and phospholipids in } \\
\text { the circulation and liver }\end{array}$ & & [135] \\
\hline \multicolumn{8}{|c|}{ Other plants-NAFLD } \\
\hline $\begin{array}{l}\text { Heshouwu } \\
\text { (Fallopia } \\
\text { multiflora) }\end{array}$ & $\begin{array}{l}\text { Stilbenes } \\
\text { anthraquinones }\end{array}$ & $\begin{array}{l}\text { In } \\
\text { vitro } \\
\text { In vivo }\end{array}$ & $\begin{array}{c}\text { L02 cell } \\
\text { Wistar rats }\end{array}$ & $\begin{array}{l}3.75,7.5,15 \\
30,60 \mu \mathrm{g} / \mathrm{mL} \\
70,140 \\
280 \mathrm{mg} / \mathrm{kg}\end{array}$ & $\begin{array}{l}\text { Improving } \\
\text { mitochondrial } \beta \\
\text { oxidation and } \\
\text { dyslipidemia }\end{array}$ & $\begin{array}{l}\text { Increasing the expression of } \\
\text { CPT- } 1 \alpha\end{array}$ & [136] \\
\hline \multirow[t]{4}{*}{$\begin{array}{l}\text { Hongjingtian } \\
\text { (Rhodiola } \\
\text { rosea) }\end{array}$} & Salidroside & $\begin{array}{l}\text { In } \\
\text { vitro }\end{array}$ & L02 cell & $\begin{array}{l}75,150 \\
300 \mu \mathrm{g} / \mathrm{mL}\end{array}$ & $\begin{array}{l}\text { Alleviating steatosis, } \\
\text { inflammation, and } \\
\text { activating autophagy }\end{array}$ & $\begin{array}{l}\text { Inhibiting the TRPM2-Ca2 } \\
\text { +-CaMKII pathway }\end{array}$ & [137] \\
\hline & Silybin & $\begin{array}{l}\text { In } \\
\text { vitro }\end{array}$ & $\mathrm{FaO}$ cells & $50 \mu \mathrm{M}$ & $\begin{array}{c}\text { Alleviating fat } \\
\text { accumulation and } \\
\text { mitochondrial damage }\end{array}$ & $\begin{array}{l}\text { Increasing the expression of } \\
\text { PPAR- } \alpha / \delta \text { and decreasing the } \\
\text { expression of PPAR- } \gamma\end{array}$ & [138] \\
\hline & Silybin & In vivo & $\begin{array}{l}\text { C57BL/6J } \\
\text { mice }\end{array}$ & $\begin{array}{c}50, \\
100 \mathrm{mg} / \mathrm{kg} / \text { day }\end{array}$ & $\begin{array}{l}\text { Regulating lipid } \\
\text { metabolism and global } \\
\text { metabolic pathways }\end{array}$ & $\begin{array}{l}\text { Modulating the metabolisms of } \\
\text { lipid, polyol, amino acid, urea } \\
\text { cycle, and TCA cycle }\end{array}$ & [139] \\
\hline & $\begin{array}{l}\text { Curcumin and } \\
\text { salidroside }\end{array}$ & In vivo & SD rats & $\begin{array}{l}21.76 \mathrm{mg} / \mathrm{kg} / \mathrm{d} \\
\text { and } \\
5.77 \mathrm{mg} / \mathrm{kg} / \mathrm{d}\end{array}$ & $\begin{array}{l}\text { Alleviating insulin } \\
\text { resistance and lipid } \\
\text { peroxidation }\end{array}$ & Activating the AMPK pathway & [140] \\
\hline $\begin{array}{l}\text { Cassia } \\
\text { (Cassia } \\
\text { obtusifolia) }\end{array}$ & & In vivo & Wistar rats & $0.5,1,2 \mathrm{~g} / \mathrm{kg}$ & $\begin{array}{c}\text { Alleviating } \\
\text { histopathological } \\
\text { changes, dyslipidemia, } \\
\text { and lipid peroxidation } \\
\text { in the liver }\end{array}$ & $\begin{array}{c}\text { Increasing the activities of SOD } \\
\text { and GSH }\end{array}$ & [141] \\
\hline $\begin{array}{l}\text { Jishiteng } \\
\text { (Paederia } \\
\text { scandens) }\end{array}$ & & In vivo & $\begin{array}{l}\text { Ross } 305 \\
\text { chicks }\end{array}$ & $2 \mathrm{mg} / \mathrm{kg}$ & $\begin{array}{l}\text { Alleviating oxidative } \\
\text { stress }\end{array}$ & Decreasing the level of HSP7C & [142] \\
\hline $\begin{array}{l}\text { Heshouwu } \\
\text { (Fallopia } \\
\text { multiflora) }\end{array}$ & & In vivo & Zebrafish & $\begin{array}{c}0.5,1 \mathrm{mg} / \mathrm{mL} \\
0.25 \\
0.5 \mu \mathrm{g} / \mathrm{mL}\end{array}$ & $\begin{array}{l}\text { Reducing lipogenesis } \\
\text { and insulin resistance }\end{array}$ & $\begin{array}{l}\text { Activating the PI3K-Akt } 2 \\
\text {-AMPK-PPAR- } \alpha \text { pathway }\end{array}$ & [143] \\
\hline \multicolumn{8}{|c|}{ Other plants-AFLD } \\
\hline & Ginsenosides & $\begin{array}{l}\text { In } \\
\text { vitro }\end{array}$ & L02 cells & $\begin{array}{c}25,50 \\
100 \mu \mathrm{g} / \mathrm{mL}\end{array}$ & $\begin{array}{l}\text { Alleviating steatosis, } \\
\text { oxidative stress, and }\end{array}$ & & [144] \\
\hline
\end{tabular}


TABLE 1: Continued.

\begin{tabular}{|c|c|c|c|c|c|c|c|}
\hline $\begin{array}{l}\text { Plant-based } \\
\text { foods }\end{array}$ & $\begin{array}{l}\text { Bioactive } \\
\text { component }\end{array}$ & $\begin{array}{l}\text { Study } \\
\text { type }\end{array}$ & Models & Doses & Main effects & Molecular mechanisms & Ref \\
\hline $\begin{array}{l}\text { Ginseng } \\
\text { (Panax } \\
\text { ginseng) }\end{array}$ & & & & & $\begin{array}{l}\text { mitochondrial } \\
\text { dysfunction }\end{array}$ & $\begin{array}{l}\text { Increasing the expression of } \\
\text { PPAR- } \alpha \text { and decreasing the } \\
\text { expression of CYP2E1 }\end{array}$ & \\
\hline $\begin{array}{l}\text { White flower } \\
\text { dandelion } \\
\text { (Taraxacum } \\
\text { coreanum) }\end{array}$ & & In vivo & $\mathrm{SD}$ rats & $1 \mathrm{~g} / \mathrm{kg} /$ day & $\begin{array}{l}\text { Improving body } \\
\text { composition, glucose } \\
\text { metabolism, ethanol } \\
\text { degradation, and gut } \\
\text { dysbiosis }\end{array}$ & $\begin{array}{l}\text { Decreasing the ratio of } \\
\text { Firmicutes to Bacteroidetes }\end{array}$ & {$[100]$} \\
\hline $\begin{array}{l}\text { Zhijuzi } \\
\text { (Hovenia } \\
\text { dulcis) }\end{array}$ & & In vivo & SD rats & $\begin{array}{c}300, \\
500 \mathrm{mg} / \mathrm{kg}\end{array}$ & $\begin{array}{l}\text { Alleviating steatosis and } \\
\text { inflammation }\end{array}$ & $\begin{array}{l}\text { Increasing PPAR- } \alpha \text {, PPAR- } \\
\gamma \mathrm{C} 1 \alpha, \mathrm{CPT}-1 \alpha \text {, and Acsl1 gene } \\
\text { expression, decreasing Myd } 88, \\
\text { TNF- } \alpha \text {, and CRP gene } \\
\text { expression }\end{array}$ & [145] \\
\hline $\begin{array}{l}\text { Platycodon } \\
\text { grandiflorum }\end{array}$ & Platycodin D & In vivo & $\mathrm{SD}$ rats & $\begin{array}{c}10,20 \\
30 \mathrm{mg} / \mathrm{kg} / \text { day }\end{array}$ & $\begin{array}{l}\text { Inhibiting inflammation } \\
\text { and endotoxic process }\end{array}$ & $\begin{array}{l}\text { Inhibiting the TLR4-MyD88- } \\
\text { NF- } \kappa \text { B pathway }\end{array}$ & [146] \\
\hline $\begin{array}{l}\text { Ecklonia } \\
\text { stolonifera }\end{array}$ & Phlorotannins & In vivo & $\mathrm{SD}$ rats & $\begin{array}{c}50,100 \\
200 \mathrm{mg} / \mathrm{kg} / \text { day }\end{array}$ & $\begin{array}{l}\text { Improving liver } \\
\text { function and lipid } \\
\text { profiles }\end{array}$ & $\begin{array}{l}\text { Increasing the expression of } \\
\text { PPAR- } \alpha \text { and CPT-1 and } \\
\text { decreasing the expression of } \\
\text { SREBP-1c }\end{array}$ & [147] \\
\hline \multicolumn{8}{|c|}{$\begin{array}{l}\text { ABCA1: ATP-binding cassette subfamily A1; ABCG1: ATP-binding cassette subfamily G1; ACC: acetyl-CoA carboxylase; ADH: alcohol dehydrogenase; Akt: } \\
\text { protein kinase B; ALT: alanine aminotransferase; AMPK: adenosine } 5^{\prime} \text {-monophosphate-activated protein kinase; ALDH: aldehyde dehydrogenase; ALP: } \\
\text { alkaline phosphatase; AST: aspartate aminotransferase; Bax: Bcl2-associated X protein; Bcl-2: B-cell lymphoma-2; CAT: catalase; CPT-1: carnitine } \\
\text { palmitoyltransferase 1; CYP2E1: cytochrome P450 2E1; EGCG: epigallocatechin gallate; FAS: fatty acid synthase; FOXO1: forkhead box protein O1; FXR: } \\
\text { farnesoid X receptor; GSH: glutathione; GSH-Px: glutathione peroxidase; GSSG: oxidized glutathione; HNF4A: hepatocyte nuclear factor } 4 \alpha \text {; IL-1 } 1 \beta / 6 / 8 \text { : } \\
\text { interleukin-1 } \beta / 6 / 8 \text {; IFN- } \gamma \text { : interferon- } \gamma \text {; LPS: lipopolysaccharide; LXR } \alpha \text { : liver X receptor } \alpha \text {; MCP-1: monocyte chemoattractant protein } 1 \text {; MDA: } \\
\text { malondialdehyde; miR-34a/194: microRNA-34a/194; mTOR: mammalian target rapamycin; MTP: microsomal triglyceride transfer protein; NADPH: } \\
\text { nicotinamide adenine dinucleotide phosphate; NF- } \alpha \text { B: nuclear factor kappa-B; Nrf2: nuclear factor erythroid 2-related factor 2; PBM cells: peripheral blood } \\
\text { mononuclear cells; PGC-1 } \alpha \text { : peroxisomal proliferator-activated receptor-gamma coactivator- } 1 \alpha \text {; PKA: protein kinase A; PPAR- } \alpha / \gamma \text { : peroxisome proliferator- } \\
\text { activated receptor- } \alpha / \gamma \text {; PTP1B: protein tyrosine phosphatase 1B; ROS: reactive oxygen species; SCD 1: stearoyl-CoA desaturase } 1 \text {; SD rats: Sprague-Dawley } \\
\text { rats; SIRT1: sirtuin 1; SOD: superoxide dismutase; SREBP-1c: sterol regulatory element-binding transcription factor } 1 \mathrm{c} \text {; STAT3: signal transducer and } \\
\text { activator of transcription 3; TG: triglyceride; TLR 4: toll-like receptor 4; TNF- } \alpha \text { : tumor necrosis factor- } \alpha \text {; VLDL: very low-density lipoprotein; ZO-1: zonula } \\
\text { occludens-1. }\end{array}$} \\
\hline
\end{tabular}

response by downregulating the NF- $\kappa \mathrm{B}$ signaling pathway [101].

In short, some fruits (such as mulberry, berries, lychee, and lemon), as well as their bioactive compounds (such as resveratrol, flavonoids, and phenolic extracts) could alleviate AFLD mainly due to the promotion of ethanol metabolism and the inhibition of liver cell apoptosis, as well as the improvement of hepatic steatosis, oxidative stress, inflammation, and gut dysbiosis (Table 1) with regulating AMPK, PPAR- $\alpha$, Nrf2, and SIRT1 signaling pathways (Figure 3).

\subsection{Spices and FLD}

\subsubsection{Mechanisms of Spices and Their Bioactive Compounds} on NAFLD. The in vitro study showed that curcumin could improve lipid accumulation by increasing the levels of CYP3A and CYP7A while decreasing the level of SREBP-1c in the primary liver cells [102]. Meanwhile, curcumin could alleviate NAFLD by decreasing the levels of lipid profiles, inhibiting O-GlcNAcylation, as well as reducing the generation of reactive oxygen species (ROS), TNF- $\alpha$, and interferon- $\gamma($ IFN- $\gamma)$ in cells $[103,104]$. An in vivo study reported that oral intake of curcumin attenuated hepatic lipid accumulation in C57BL/6 mice by upregulating the expres- sion of Nrf2 and farnesoid X receptor (FXR) while downregulating the expression of liver X receptor $\alpha(\mathrm{LXR} \alpha)$ [102]. Also, curcumin alleviated hepatic lipid accumulation and inflammation in C57BL/6J mice by upregulating the SIRT1AMPK-acetyl-CoA carboxylase (ACC) signaling pathway and inhibiting the O-GlcNAcylation of NF- $\kappa$ B [104]. Besides, recent studies also indicated that onion significantly ameliorated hepatic steatosis, ballooning, and lobular and portal inflammation in SD rats and decreased the serum levels of alanine aminotransferase (ALT), aspartate aminotransferase (AST), TG, insulin, glucose, and the level of TNF- $\alpha$ in hepatocytes $[105,106]$. Collectively, spices like onion and curcumin from turmeric could prevent NAFLD through improving lipid metabolism, oxidative stress, and inflammation (Table 1 ) by regulating SIRT1, NF- $\kappa \mathrm{B}$, and Nrf2-FXRLXR $\alpha$ signaling pathways (Figure 2).

4.2.2. Mechanisms of Spices and Their Bioactive Compounds on AFLD. Bioactive compounds from spices have been reported to fight against AFLD. Allicin, a bioactive compound from garlic, could attenuate AFLD in C57BL/6 mice by increasing the levels of glutathione (GSH), CAT, and the activity of alcohol dehydrogenase $(\mathrm{ADH})$, as well as decreasing the levels of SERBP-1c, CYP2E1, TNF- $\alpha$, and interleukins 


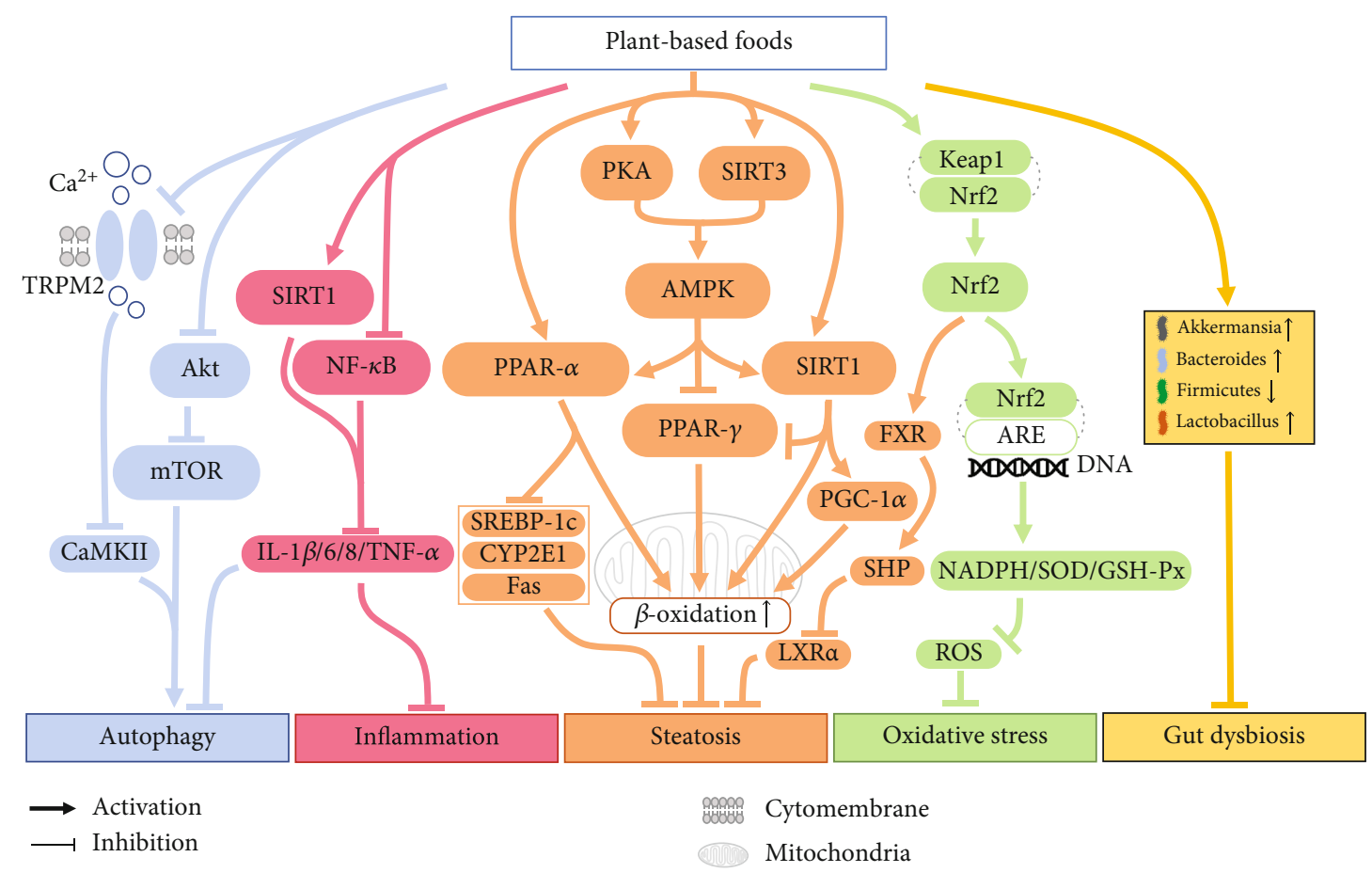

FIgURE 2: The molecular mechanisms of plant-based foods in the alleviation of NAFLD. The major molecular mechanisms include the alleviation of hepatic steatosis, oxidative stress, inflammation, gut dysbiosis, and the regulation of autophagy. To be specific, fruits, spices, and teas alleviated hepatic steatosis by activating AMPK, PPAR- $\alpha$, SIRT1, and FXR and inhibiting PPAR- $\gamma$ pathways. Besides, the antioxidative stress property of such plants was mainly associated with the activation of the Nrf2 pathway. Moreover, plant-based foods could alleviate inflammation by activating SIRT1, while inhibiting NF- $\kappa$ B pathways. Furthermore, these plants alleviated gut dysbiosis by regulating the abundance of Akkermansia, Bacteroides, Firmicutes, and Lactobacillus. Also, such plants could regulate autophagy by inhibiting the Akt pathway and reducing the production of autophagic biomarkers. Akt: protein kinase B; AMPK: adenosine $5^{\prime}$ -monophosphate-activated protein kinase; ARE: Nrf2-antioxidant response element; CYP2E1: cytochrome P450 2E1; FAS: fatty acid synthase; FXR: farnesoid X receptor; IL-1 $\beta / 6 / 8$ : interleukin-1 $\beta / 6 / 8$; LXR $\alpha$ : liver X receptor $\alpha$; mTOR: mammalian target rapamycin; NF$\kappa \mathrm{B}$ : nuclear factor kappa-B; Nrf2: nuclear factor erythroid 2-related factor 2; PGC-1 $\alpha$ : peroxisomal proliferator-activated receptor-gamma coactivator- $1 \alpha$; PKA: protein kinase A; PPAR- $\alpha / \gamma$ : peroxisome proliferator-activated receptor- $\alpha / \gamma$; ROS: reactive oxygen species; SIRT1: sirtuin 1; SOD: superoxide dismutase; SREBP-1c: sterol regulatory element-binding transcription factor 1c; TNF- $\alpha$ : tumor necrosis factor- $\alpha$.

[107]. Also, allicin could alleviate alcohol-induced hepatic steatosis in C57BL/6 mice by modifying gut dysbiosis and then reducing the production of lipopolysaccharide (LPS) and further inhibiting TLR4-mediated inflammation [62]. Besides, the consumption of the ginger extract could significantly regulate lipid homeostasis in Wistar rats by increasing the expression of hepatocyte nuclear factor $4 \alpha$ gene (HNF4A), while decreasing the expression of the protein tyrosine phosphatase $1 B$ gene $(P T P 1 B)[108,148]$. Moreover, in a mouse model of AFLD, oral gavage of curcumin could suppress the biosynthesis of fatty acids, the pathway of pentose glucuronate, and the metabolism of glyoxylate, dicarboxylate, and pyruvate [109]. To sum up, several spices (like garlic and ginger) and their bioactive compounds (like allicin) could alleviate lipid accumulation, oxidative stress, inflammation, and gut dysbiosis (Table 1), with increasing activity of $\mathrm{ADH}$ and the levels of antioxidant enzymes, suppressing fatty acid biosynthesis and TLR4 signaling pathway, and regulating the expressions of HNF4A and PTP1B genes (Figure 3).

\subsection{Teas and FLD}

4.3.1. Mechanisms of Teas and Their Bioactive Compounds on NAFLD. Tea is one of the most popular beverages in the world and possesses hepatoprotective effects based on in vitro studies. The aqueous extract of green tea could moderate NAFLD in HepG2 cells by inhibiting the activity of TNF- $\alpha$ and further downregulating microRNA-34a (miR-34a) and upregulating miR-194 [110]. Besides, raw bowl tea (also called Tuocha) is a type of dark tea, and the polyphenols extracted from raw bowl tea could inhibit the proliferation of 3T3-L1 preadipocytes, suggesting its beneficial effect to alleviate NAFLD [111]. The aqueous extract of Hao Ling tea, which is a blend of green, oolong, and pu-erh tea, could inhibit the production of mitochondrial ROS in the NAFLD cell model [112]. Tea bioactive compounds also exhibit protective effects on NAFLD in vivo. The aqueous extract of green tea alleviated NAFLD in C57BL/6 mice by modulating the expression of miR-34a and miR-194, resulting in increasing the expression of SIRT1, PPAR- $\alpha$, and insulininduced gene 2 (Insig2), as well as decreasing the expression of apolipoprotein a5, 3-hydroxy-3-methyl glutaryl coenzyme A synthase (HMG-CoA synthase), and HMG-CoA reductase [110]. Also, the consumption of the green tea extract alleviated hyperlipidemia and enhanced the activity of superoxide 


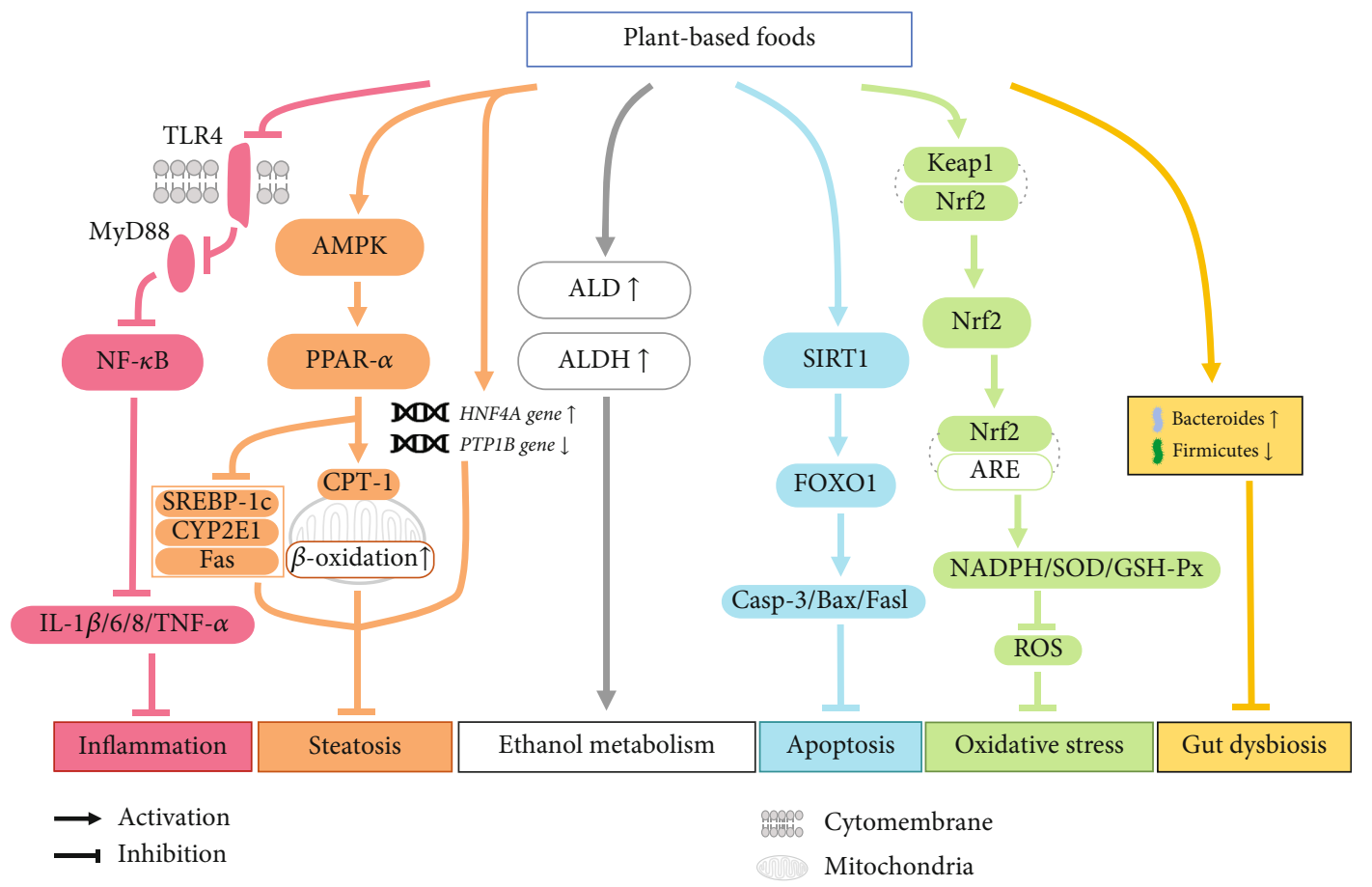

FIGURE 3: The molecular mechanisms of plant-based foods in the alleviation of AFLD. The major molecular mechanisms include the promotion of ethanol metabolism and inhibition of apoptosis, hepatic steatosis, oxidative stress, inflammation, and gut dysbiosis. First of all, plant-based foods accelerated ethanol metabolism by increasing the activities of ALD and ALDH. Also, these plants inhibited apoptosis by activating the SIRT1 pathway. Moreover, they could alleviate hepatic steatosis by activating the AMPK-PPAR- $\alpha$ pathway and regulating lipid homeostasis-related genes (like HNF4A and PTP1B). Furthermore, the antioxidative effect of such plants was related to the upregulation of the Nrf2 pathway. Besides, edible plants ameliorated inflammation by inhibiting TLR4 and NF- $\kappa$ B pathways. Additionally, these plants attenuated gut dysbiosis by regulating the abundance of Bacteroides and Firmicutes. AMPK: adenosine 5 ' -monophosphate-activated protein kinase; ARE: Nrf2-antioxidant response element; CPT-1: carnitine palmitoyltransferase 1; CYP2E1: cytochrome P450 2E1; FAS: fatty acid synthase; IL-1 $\beta / 6 / 8$ : interleukin-1 $\beta / 6 / 8$; NF- $\kappa$ B: nuclear factor kappa-B; Nrf2: nuclear factor erythroid 2-related factor 2; PPAR- $\alpha$ : peroxisome proliferator-activated receptor- $\alpha$; ROS: reactive oxygen species; SOD: superoxide dismutase; SREBP-1c: sterol regulatory element-binding transcription factor 1c; TNF- $\alpha$ : tumor necrosis factor- $\alpha$.

dismutase (SOD) in Wistar rats [113]. Moreover, oral gavage of green tea polyphenols could inhibit the hepatic lipogenesis in Zucker rats by upregulating the AMPK signaling pathway, with reducing the levels of insulin, glucose, ALT, AST, TNF- $\alpha$, and interleukin-6 (IL-6) [114]. Similarly, polyphenols from raw bowl tea could decrease the serum levels of lipid profiles and inflammatory cytokines in C57BL/6N mice [111]. More importantly, polyphenols from raw bowl tea were beneficial to alleviating NAFLD in C57BL/6N mice by regulating the abundance of gut microbiota, such as decreasing the level of Firmicutes and increasing the levels of Bacteroides and Akkermansia [111]. Besides, the extract of Ning Hong black tea decreased the number of hepatic lipid droplets in SD rats by upregulating the expression of PPAR- $\alpha$ and microsomal triglyceride transfer protein, as well as promoting fatty acid $\beta$ oxidation [115].

In short, green, black, and dark teas, as well as tea polyphenols, could alleviate NAFLD through improving liver function, regulating glucose and lipid metabolism, decreasing the levels of ROS and inflammatory cytokines, and modulating the composition of gut microbiota (Table 1), with decreasing the expression of $\mathrm{miR}-34 \mathrm{a}$, increasing the expression of miR-194, and upregulating SIRT1, PPAR- $\alpha$, and AMPK signaling pathways (Figure 2).
4.3.2. Mechanisms of Teas and Their Bioactive Compounds on $A F L D$. It has been reported that several types of teas play a key role in alcohol metabolism [149, 150]. Green tea could accelerate ethanol metabolism in Kunming mice by increasing the activities of $\mathrm{ADH}$ and aldehyde dehydrogenase (ALDH), thus exerting a hepatoprotective effect [116]. Also, the consumption of green tea and its polyphenolic extract could attenuate ALFD in Wistar rats by ameliorating oxidative stress and necrosis $[117,118]$. The molecular mechanisms of green tea against AFLD were due to decreasing the levels of TG, ALT, and ROS and inhibiting the expression of SREBP-1c, FAS, and CYP2E1 [117, 118]. EGCG is one of the major polyphenols in green tea, and it could alleviate ethanol-induced AFLD in Wistar rats by promoting the phosphorylation of ACC and increasing the level of CPT-1 $[119,151]$. Furthermore, catechins extracted from green tea could alleviate alcohol-induced fatty changes, liver dysfunction, oxidative stress, and inflammation in Wistar rats by suppressing the NF- $\kappa \mathrm{B}$ signaling pathway [120].

Collectively, green tea and its bioactive compounds (like EGCG) could alleviate AFLD by improving ethanol metabolism, lipid metabolism, oxidative stress, inflammation, and necrosis (Table 1), with increasing the activities of $\mathrm{ADH}$ and ALDH and the phosphorylation of ACC, reducing the 
expression of SREBP-1c, FAS, and CYP2E1, and inhibiting the NF- $\kappa \mathrm{B}$ signaling pathway (Figure 3 ).

\subsection{Coffee and FLD}

4.4.1. Mechanisms of Coffee and Its Bioactive Compounds on NAFLD. Coffee and its bioactive compounds were widely investigated $[152,153]$. The in vitro study showed that caffeic acid could ameliorate hepatic steatosis and decrease endoplasmic reticulum stress by increasing autophagy in AML12 cells [121]. Similarly, trigonelline is one of the main components of coffee and could prevent hepatic lipid accumulation and lipotoxicity in AML 12 cells and HepG2 cells by promoting autophagy [122]. Besides, caffeine could also modulate hepatocyte steatosis by activating SIRT3 and AMPK in oleate-treated HepG2 cells [123].

The in vivo study showed that coffee consumption played a role in the prevention of NAFLD [124]. The coffee pulp aqueous extract improved hepatic steatosis, insulin resistance, and oxidative stress in Wistar rats by upregulating the expression of PPAR- $\alpha$; meanwhile, the combined administration of the extract and simvastatin could additively suppress the expression of PPAR- $\gamma$ and SREBP-1c [125]. Moreover, coffee supplementation could alleviate hepatic fat deposition and metabolic derangement in NAFLD mice by improving liver fat oxidation, intestinal cholesterol efflux, energy metabolism, and gut permeability [126]. Furthermore, trigonelline from coffee could ameliorate NAFLD in $\mathrm{SD}$ rats by increasing the expression of $\mathrm{Bcl}-2$ protein and decreasing the expression of Bax protein in the liver [127]. Caffeine was another important bioactive compound in coffee and could suppress hepatic steatosis by activating SIRT3-AMPK-ACC and signal transducer and activator of transcription 3 (STAT3) pathways [123, 128]. Moreover, the green coffee extract showed more effectiveness in reducing hepatic TG than caffeine $[129,130]$. Furthermore, decaffeinated coffee could revert to normal function the gut permeability and intestinal barrier function by increasing the expression of tight junction proteins and decreasing TLR4 expression [131]. It is worth noting that prenatal caffeine exposure of pregnant rats could increase the susceptibility of NAFLD in offspring [132].

In summary, some studies reported that coffee and its bioactive compounds (like caffeic acid, trigonelline, and caffeine) could be beneficial for NAFLD prevention through improving hepatic steatosis, insulin resistance, oxidative stress, gut permeability, and autophagy (Table 1), with regulating phosphorylation of mTOR and the expression of PPAR- $\alpha / \gamma$, SREBP-1c, and TLR4, as well as activating the SIRT3-AMPK-ACC signaling pathway (Figure 2). But several studies showed opposite results, therefore, the hepatoprotective effects and mechanisms of coffee and caffeine still need further investigation.

4.4.2. Mechanisms of Coffee and Its Bioactive Compounds on AFLD. Since caffeine possesses a strong association with liver function, several studies discussed the bioactive effect of caffeine on fat metabolism in the liver under alcohol intake [133-135]. Oral administration of caffeine could attenuate alcohol-induced hepatic cell damage, steatosis, and inflammatory response in Kunming mice by decreasing the expression of lipogenic genes and the levels of inflammatory cytokines in the serum and liver [133]. Further study showed that caffeine could even inhibit the activation of the hepatic stellate cell, thus beneficial to preventing alcohol-induced liver fibrosis in SD rats [134]. Besides, oral administration of caffeic acid to alcohol-fed Wistar rats decreased the levels of TG, total cholesterol (TC), free fatty acids, and phospholipids in circulation and the liver [135]. Collectively, caffeine and caffeic acid could ameliorate AFLD through attenuating hepatocyte damage, hepatic steatosis, and inflammatory response (Table 1), accompanied by regulating the expression of lipogenic genes and the levels of inflammatory cytokines (Figure 3).

\subsection{Other Plants and FLD}

4.5.1. Mechanisms of Other Plants and Their Bioactive Compounds on NAFLD. It is well known that plenty of medicinal plants are edible foods, which have been developed into functional foods with hepatoprotective function $[154,155]$. An in vitro study revealed that Heshouwu (Fallopia multiflora) could alleviate NAFLD by promoting mitochondrial $\beta$ oxidation and attenuating lipid accumulation in L02 human liver cells [136]. Moreover, salidroside, a phenylpropanoid glycoside compound from Hongjingtian (Rhodiola rosea), could ameliorate NAFLD in L02 cells through alleviating steatosis and inflammation, as well as activating autophagy by downregulating the transient receptor potential melastatin2-Ca ${ }^{2+}$-calmodulin-stimulated protein kinase II (TRPM2-Ca ${ }^{2+}$-CaMKII) signaling pathway [137]. Furthermore, the treatment of silybin could alleviate NAFLD in $\mathrm{FaO}$ liver cells by increasing the expression of PPAR $-\alpha / \delta$ and decreasing the expression of PPAR- $\gamma$ [138].

The in vivo studies revealed that oral gavage of silymarin and silybin could ameliorate hepatic steatosis by regulating lipid metabolism and oxidative stress in C57BL/6J mice [139]. Besides, coadministration of salidroside and curcumin could alleviate high-fat diet-induced NAFLD in SD rats by activating the AMPK signaling pathway [140]. Similarly, the intake of the ethanol extract of Cassia semen (Cassia obtusifolia) could decrease the levels of liver enzymes, TG, TC, SOD, TNF- $\alpha$, and interleukins in Wistar rats [141]. Furthermore, the consumption of Jishiteng (Paederia scandens) could reduce hepatic ROS and MDA levels by decreasing the level of heat shock cognate $71 \mathrm{kDa}$ protein (HSP7C) in a chicken model of NAFLD [142].

Generally, several medicinal plants and their bioactive compounds could significantly alleviate NAFLD by regulating lipid metabolism, oxidative stress, and inflammation (Table 1). The underlying molecular mechanisms mainly included the upregulation of AMPK, Nrf2, SIRT1, and SIRT3 signaling pathways (Figure 2).

4.5.2. Mechanisms of Other Plants and Their Bioactive Compounds on AFLD. According to recent experimental 
studies, some medicinal plants could be potential agents for the prevention of AFLD [144-146]. The in vitro study showed that the treatment of ginsenosides extracted from ginseng (Panax ginseng) could alleviate ethanol-induced hepatocyte steatosis, oxidative stress, and mitochondrial dysfunction in L02 cells by increasing the expression of PPAR- $\alpha$ and decreasing the expression of CYP2E1 [144].

The in vivo study showed that the intake of the aqueous extract of white flower dandelion (Taraxacum coreanum) ameliorated AFLD in SD rats by improving ethanol degradation, glucose metabolism, and composition of gut microbiota [100]. Besides, Zhijuzi (Hovenia dulcis) could alleviate hepatic steatosis and inflammation in the AFLD rat model by upregulating the expressions of PPAR- $\alpha$, CPT- $1 \alpha$, and long-chain fatty acid CoA ligase 1 (Acsl1), as well as downregulating the expressions of myeloid differentiation factor 88 (Myd88), TNF- $\alpha$, and C-reactive protein (CRP) [145]. Moreover, platycodin D which was extracted from Jiegeng (Platycodon grandiflorum) ameliorated AFLD in SD rats by inhibiting the TLR4-Myd88-NF- $\kappa \mathrm{B}$ signaling pathway [146]. Furthermore, Ecklonia stolonifera is an edible perennial brown marine alga belonging to the family Laminariaceae and could improve lipid metabolism in the AFLD rat model by increasing the expression of PPAR- $\alpha$ and CPT- 1 , accompanied by decreasing the serum levels of ALT, AST, and the hepatic level of MDA and SREBP-1c [147]

In conclusion, medicinal plants and their bioactive compounds could alleviate AFLD due to their properties of improving ethanol degradation, regulating lipid and glucose metabolism, and alleviating inflammation and gut dysbiosis (Table 1). The underlying molecular mechanisms included the upregulation of AMPK, PPAR- $\alpha$, and ACC signaling pathways, as well as the downregulation of TLR4, Myd88, and NF- $\kappa$ B signaling pathways (Figure 3 ).

\section{Clinical Trials of Plant-Based Foods and Their Bioactive Compounds}

Several clinical trials have proceeded in recent years to better verify the effects of plant-based foods, such as fruits, spices, and teas on NAFLD. A randomized cross-over clinical trial based on 61 obese children with NAFLD proved that daily consumption of $100 \mathrm{~mL}$ of tomato juice for 60 days could improve hepatic steatosis, insulin resistance, levels of leptin, and lipid profiles [156]. Additionally, a clinical trial found that in 55 patients, a 24 -week intake of $36 \mathrm{~g}$ per day of dried grapes was beneficial for the prevention and management of NAFLD [157]. Moreover, the consumption of 30 to $35 \mathrm{~g}$ per day of dietary fiber from fruits could alleviate hepatic steatosis and improve intestinal permeability in NAFLD patients [158].

Spices and their bioactive compounds are also beneficial to FLD patients. Several double-blind randomized placebocontrolled clinical trials showed that the intake of $500 \mathrm{mg}$ of green cardamom 3 times per day for 3 months could decrease the degree of fatty liver in NAFLD patients, accompanied by decreasing the levels of ALT, CRP, TNF- $\alpha$, and IL-6, as well as increasing the serum level of SIRT1 [159]. Besides, the intake of $70 \mathrm{mg}$ per day of curcumin for 8 weeks significantly reduced fatty liver in NAFLD patients, accompanied by improving the serum levels of liver enzymes, lipid profiles, glucose, and glycated hemoglobin [160]. Moreover, a clinical trial based on 55 subjects demonstrated that the supplementation of $500 \mathrm{mg}$ per day of curcumin decreased the serum levels of inflammatory cytokines (like TNF- $\alpha$ and interleukins) in NAFLD patients [161]. Furthermore, a randomized, double-blind, placebo-controlled study based on 58 NAFLD patients showed that curcumin could alleviate NAFLD by regulating amino acid metabolism, tricarboxylic acid cycle, bile acid metabolism, and gut microbiota [162].

Clinical trials also indicate that oral intake of green tea shows protective effects against FLD. Oral intake of $500 \mathrm{mg}$ green tea tablet containing $50 \mathrm{mg}$ of standardized total polyphenols 3 times per day for 3 months effectively decreased hepatic fat accumulation, alleviated fatty liver grade, and improved liver function in 52 NAFLD patients aged 10 to 16 years [163]. Additionally, the intake of $500 \mathrm{mg}$ of the green tea extract for 3 months could improve the serum levels of ALT, AST, lipid profiles, and inflammatory markers in NAFLD patients [164]. Moreover, a meta-analysis including 15 randomized clinical trials further confirmed the hepatoprotective effect of green tea and catechins against NAFLD [165].

A clinical trial with 48 NAFLD patients demonstrated that a daily dose consumption of $400 \mathrm{mg}$ green coffee extract for 8 weeks could improve fasting blood glucose (mean difference (MD): -11.50 ; 95\%CI: $-19.59,-3.42$ ), insulin resistance status (MD: $-0.97 ; 95 \% \mathrm{CI}:-1.84,-0.11$ ), body weight (MD: -1.73 ; $95 \%$ CI: $-2.44,-1.01$ ), body mass index (BMI) (MD: -0.57 ; 95\%CI: $-0.84,-0.29$ ), and waist circumference (MD: -3.69 ; 95\% CI: $-5.85,-1.54$ ), as well as increasing the serum level of leptin [166]. Also, the consumption of the green coffee extract increased the serum level of highdensity lipoprotein cholesterol (HDL-C) (MD: 7.06; 95\%CI: $0.25,13.87)$ [167]. Moreover, a randomized, placebo-controlled, clinical trial with 26 NAFLD patients showed that the coadministration of $200 \mathrm{mg}$ caffeine and chlorogenic acid for 12 weeks could more effectively decrease body weight than caffeine consumption alone [168]. Furthermore, a meta-analysis showed that although total caffeine intake was not related to the prevalence or progression of NAFLD, the regular intake of caffeine from daily coffee consumption could reduce the risk of hepatic fibrosis in NAFLD patients [169].

Other plant-based foods, like whole grain, also possessed a beneficial effect to alleviate NAFLD. An open-label, randomized controlled clinical trial with 112 NAFLD patients demonstrated that the consumption of whole grain for 12 weeks could alleviate hepatic steatosis and liver dysfunction [170]. Similarly, a randomized, double-blinded, parallelarmed study with 40 NAFLD patients indicated that the consumption of ancient Khorasan wheat (Triticum turgidum) for 3 months could reduce the levels of ALT, AST, ALP, TNF- $\alpha$, IL-8, and IFN- $\gamma$ [171]. Moreover, black seed (Nigella sativa) was a kind of edible and medicinal plant that belongs to the Ranunculaceae family, and it was very popular among Iranians $[172,173]$. The consumption of $2 \mathrm{~g}$ per day of black seed (Nigella sativa) for 3 months could ameliorate hepatic 
steatosis in NAFLD patients [174]. Furthermore, a 3-month intake of 40 to $60 \mathrm{~g}$ per day of caper fruit (Capparis spinosa) pickle, a traditional food widely found in the western or central regions of Asia, significantly reduced the serum levels of AST and ALT and improved lipid profiles in NAFLD patients $[175,176]$. In addition, a random-controlled clinical trial including 100 NAFLD patients demonstrated that a combined intake of $30 \mathrm{~g}$ flaxseed and $1 \mathrm{~g}$ hesperidin for 12 weeks could improve glucose and lipid metabolism and reduce hepatic steatosis and inflammation [177].

In short, clinical trials confirmed that the consumption of fruits (such as tomato and grapes), spices (like green cardamom), teas (mainly green tea), coffee, and other plantbased foods (like whole grain, black seed, and caper), as well as their bioactive compounds (such as dietary fiber and curcumin), could alleviate NAFLD in human.

\section{Safety and Application Issues of Plant-Based Foods and Their Bioactive Compounds in Patients}

The plant-based, high-fiber, and low-fat diet has been recognized as a healthy lifestyle and recommended to manage FLD for a long time. It is very necessary to evaluate the safety and application issues of plant-based foods and their bioactive compounds.

6.1. Safety and Application Issue of Fruits and Their Bioactive Compounds. The pharmacokinetic study revealed that after ingesting $500 \mathrm{mg}$ of resveratrol for 24 hours, there were no adverse reactions for all healthy volunteers, and the maximum concentration and the area under the curve of resveratrol were lower when compared with its metabolites in plasma [178]. Besides, a double-blind, randomized, placebo-controlled trial based on 32 overweight senior citizens (mean age: $73 \pm 7$ years) showed that the daily consumption of 300 or $1,000 \mathrm{mg}$ resveratrol for 90 days did not adversely affect blood chemistries [179]. It was worth noting that a clinical trial showed that the daily intake of 3,000 mg resveratrol in overweight or obese men with NAFLD for 8 weeks did not improve any features of NAFLD but increased the levels of liver enzymes [180].

Berries are recognized as healthy foods and rich in anthocyanins [181]. The in vivo study revealed that the acute oral median lethal dose of the mixture extract from common edible berries was higher than $5 \mathrm{~g} / \mathrm{kg}$ in SD rats, which means that the mixture extract of berries was a relatively safe food [182]. To further assess the safety of anthocyanin-rich fruit consumption in human, a pilot study tested the biochemical changes in blood after ingesting sweet cherries at the dose of three cups per day for 4 weeks, and it turned out that 37 overweight senior citizens were well tolerated to the intervention [183]. Moreover, 8-week treatment with $200 \mathrm{mg}$ of acylated anthocyanin from purple sweet potato twice a day in NAFLD patients could reduce the levels of liver enzymes, especially $\gamma$ glutamyl transpeptidase [184]. Furthermore, a CONSORTcompliant, randomized, double-blind, placebo-controlled pilot trial showed that the consumption of anthocyanin derived from bilberry and black currant at a dose of $320 \mathrm{mg}$ per day for 12 weeks attenuated clinical symptoms in 74 NAFLD patients without side effect reported [185].

$\mathrm{Z}$-isomers of lycopene are abundant in processed tomato products (like tomato oleoresin) with high bioavailability, and the median lethal dose of tomato oleoresin was more than $5,000 \mathrm{mg} / \mathrm{kg}$ (which was equal to $361 \mathrm{mg} / \mathrm{kg}$ of (Z)-lycopene) via a single-dose oral test in Wistar rats [186]. Further, in a repeated-dose toxicity test, after oral administrating tomato oleoresin at a dose of $4,500 \mathrm{mg} / \mathrm{kg}$ per day (which was equal to $325 \mathrm{mg} / \mathrm{kg} /$ day of (Z)-lycopene) for 4 weeks, the Wistar rats showed no adverse changes [186]. Only a few studies tried to use lycopene as a potential treatment against FLD with no adverse effects reported so that more evidence is needed on the topic [156].

The intake of fructose is recognized as a risk factor for NAFLD, and some types of fruit are fructose-rich foods [187]. A case report of a 33-year-old male with insulinoma and hypoglycemia for 4 years showed that overeating of fruits, especially the fruits rich in fructose, might exacerbate histological changes of NAFLD [188]. Therefore, the dietary recommendations of fruit intake for the fatty liver population need to be considered thoroughly and carefully.

6.2. Safety and Application Issue of Spices and Their Bioactive Compounds. A randomized controlled trial reported that NAFLD patients who received curcumin at a dose of $1,000 \mathrm{mg}$ per day for 8 weeks revealed a reduction of the body mass index, waist circumference, and the levels of liver enzymes with good tolerance [189]. Moreover, an 8-week single-arm trial on 36 NAFLD patients showed that the consumption of the curcumin-phospholipid complex at a dose of 1,500 mg per day could ameliorate NAFLD severity and none of the severe adverse events were reported [190]. Besides, a clinical trial based on 98 NAFLD patients showed that the intake of $800 \mathrm{mg}$ garlic for 15 weeks could improve hepatic steatosis compared with the placebo group (RR: 5.6; 95\%CI: $2.17,14.5$ ) with no serious adverse effects [191]. Allicin is a common phytochemical from garlic, and a previous high-quality article summarized the safety assessment of allicin application and emphasized that the highdose consumption of allicin ( 30 to $59 \mathrm{mg}$ ) could cause stomach irritation, especially when people were in a fasting state or ingested too fast [192].

\subsection{Safety and Application Issue of Teas and Their Bioactive} Compounds. Tea polyphenol and its major component EGCG are commonly recognized as healthy and safe foods $[193,194]$. A double-blind randomized clinical trial on 45 NAFLD patients showed that daily received $550 \mathrm{mg}$ of green tea tablets for 3 months significantly improved the body mass index, level of AST, and fasting blood sugar [195]. Even though green tea was famous for its therapeutic effects on NAFLD, it is still needed to be cautious about its side effect [196]. A 4-week clinical trial on 40 healthy participants found out that the intake of EGCG at a dose of 400 or $800 \mathrm{mg}$ once per day led to a few mild adverse events, such as excess gas, upset stomach, nausea, heartburn, stomach ache, abdominal pain, dizziness, headache, and muscle pain [197]. Meanwhile, the consumption of EGCG at $800 \mathrm{mg}$ per day could 
significantly increase the systemic availability of free EGCG by more than $60 \%$ [197].

6.4. Safety and Application Issue of Coffee and Its Bioactive Compounds. The coffee fruit contains a series of bioactive compounds, such as phenolic acids, methylxanthines, chlorogenic acids, and caffeine, which can be used as active ingredients of functional foods or drugs [198]. In the acute test, the oral administration of the hydroethanolic extract from green coffee fruit at a dose of $1,000 \mathrm{mg} / \mathrm{kg}$ for 14 days did not show adverse effects on mice, and the oral median lethal dose of the extract was $5,000 \mathrm{mg} / \mathrm{kg}$ per day [198]. Besides, the increase in workout time in daily life has been recognized as a healthy lifestyle and beneficial to FLD management, and caffeine is a common food additive used in preworkout supplements [199]. A double-blinded, placebocontrolled study with 17 males reported that the daily consumption of caffeine $20 \mathrm{~min}$ before exercise at a dose of $300 \mathrm{mg}$ for 28 days showed no adverse changes on the biomarkers of renal and liver functions [199]. Moreover, the oral administration of green coffee oil rich in cafestol and kahweol did not lead to adverse effects in rats based on the acute toxicity test and the subacute toxicity test [200]. Furthermore, an in vivo study reported that spent coffee grounds did not show mycotoxins and toxicity based on the pilot repeated intake study with Wistar rats and could be a sustainable and safe ingredient for preventing hepatic steatosis [201].

6.5. Safety and Application Issue of Other Plants and Their Bioactive Compounds. According to the results of a multicenter phase II clinical trial, 78 patients with nonalcoholic steatohepatitis received silymarin at a dose of 420 or $700 \mathrm{mg}$ for 48 weeks in which we did not observe significant adverse events, indicating that a higher than customary doses of silymarin treatment were safe and well tolerated [202]. Besides, salidroside, a bioactive component extracted from Hongjingtian (Rhodiola rosea), was evaluated as genotoxicity free via the reverse mutation assay, chromosomal aberration assay, and mouse micronucleus assay [203]. In addition, ginsenosides are bioactive compounds from ginseng (Panax ginseng) with low absorption and bioavailability, which made the clinical application of ginsenosides became very difficult; therefore, further studies are needed to develop the advanced delivery systems to enhance the clinical usefulness of ginsenosides [77].

To sum up, although the current mainstream view and study results indicate that increasing the proportion of plant-based food intake in the dietary diet is overall beneficial to the prevention and management of FLD, there are still some issues that remain to be resolved in the practical application. It is pleasant to notice that more and more clinical trials can further explore the new evidence of the safety and efficacy of plant-based foods and their bioactive compounds on FLD [204, 205].

\section{Conclusions}

In conclusion, millions of people suffered from FLD (including NAFLD and AFLD) and the numbers are still climbing year by year, despite the vigorous promotion of more exer- cise, weight control, and alcohol abstinence. Epidemiological evidence suggests that a healthy dietary pattern with increasing the intake of several plant-based foods could lower the risk of FLD. Further experimental studies discovered that the mechanisms of plant-based foods and their bioactive compounds against FLD included the improvement of hepatic steatosis, oxidative stress, inflammation, gut dysbiosis, apoptosis, autophagy, and ethanol metabolism. Moreover, the upregulation of AMPK, SIRT1, Nrf2, and PPAR- $\alpha$ signaling pathways and the downregulation of PPAR- $\gamma$, NF- $\kappa \mathrm{B}, \mathrm{mTOR}, \mathrm{Akt}$, and TLR4 signaling pathways played a crucial role in the remission of FLD. More importantly, since the absence of standardized therapies for FLD, a hypocaloric diet accompanied by physical activity is recommended to manage fatty liver as reported in the international guidelines. Several clinical trials confirmed the protective effects of plant-based foods against FLD. In this context, increased consumption of plant-based foods in daily life can be efficient to treat FLD patients. Furthermore, the practical application of plant-based foods and their bioactive compounds in FLD patients is well tolerated, but the safe and effective dose needs to be further confirmed. In the future, more plant-based foods need to be investigated as potentially functional foods on FLD prevention and their main bioactive compounds need to be separated and identified.

\section{Conflicts of Interest}

The authors declare that there is no conflict of interest regarding the publication of this paper.

\section{Acknowledgments}

We thank Mu-Ke Han for her assistance. This research was funded by the National Key R\&D Program of China (No. 2018YFC1604405), the Central Public-interest Scientific Institution Basal Research Fund (No. Y2020XK05), the Local Financial Funds of National Agricultural Science and Technology Center, Chengdu (No. NASC2020KR02), and the Key Project of Guangdong Provincial Science and Technology Program (No. 2014B020205002).

\section{References}

[1] S. Mitra, A. De, and A. Chowdhury, "Epidemiology of nonalcoholic and alcoholic fatty liver diseases," Translational Gastroenterology and Hepatology, vol. 5, p. 16, 2020.

[2] J. Zhou, F. Zhou, W. Wang et al., "Epidemiological features of NAFLD from 1999 to 2018 in China," Hepatology, vol. 71, no. 5, pp. 1851-1864, 2020.

[3] Q. Ye, B. Zou, Y. H. Yeo et al., "Global prevalence, incidence, and outcomes of non-obese or lean non-alcoholic fatty liver disease: a systematic review and meta-analysis," The Lancet Gastroenterology \& Hepatology, vol. 5, no. 8, pp. 739-752, 2020.

[4] F. Zhou, J. Zhou, W. Wang et al., "Unexpected rapid increase in the burden of NAFLD in China from 2008 to 2018: a systematic review and meta-analysis," Hepatology, vol. 70, no. 4, pp. 1119-1133, 2019. 
[5] S. Y. Kang, Y. J. Kim, and H. S. Park, "Trends in the prevalence of non-alcoholic fatty liver disease and its future predictions in Korean men, 1998-2035," Journal of Clinical Medicine, vol. 9, no. 8, article 2626, 2020.

[6] T. Wong, K. Dang, S. Ladhani, A. K. Singal, and R. J. Wong, "Prevalence of alcoholic fatty liver disease among adults in the United States, 2001-2016," Jama-Journal of the American Medical Association, vol. 321, no. 17, pp. 1723-1725, 2019.

[7] M. Hamaguchi, A. Obora, T. Okamura, Y. Hashimoto, T. Kojima, and M. Fukui, "Changes in metabolic complications in patients with alcoholic fatty liver disease monitored over two decades: NAGALA study," BMJ Open Gastroenterology, vol. 7, no. 1, article e000359, 2020.

[8] J. Xiao, C. T. Ho, E. C. Liong et al., "Epigallocatechin gallate attenuates fibrosis, oxidative stress, and inflammation in non-alcoholic fatty liver disease rat model through TGF/SMAD, PI3 K/Akt/FoxO1, and NF-kappa B pathways," European Journal of Nutrition, vol. 53, no. 1, pp. 187-199, 2014.

[9] A. Rietman, D. Sluik, E. J. M. Feskens, F. J. Kok, and M. Mensink, "Associations between dietary factors and markers of NAFLD in a general Dutch adult population," European Journal of Clinical Nutrition, vol. 72, no. 1, pp. 117-123, 2018.

[10] X. Feng, W. Yu, X. Li et al., "Apigenin, a modulator of PPAR $\gamma$, attenuates HFD-induced NAFLD by regulating hepatocyte lipid metabolism and oxidative stress via Nrf2 activation," Biochemical Pharmacology, vol. 136, pp. 136149, 2017.

[11] N. Katsiki, D. P. Mikhailidis, and C. S. Mantzoros, "Nonalcoholic fatty liver disease and dyslipidemia: an update," Metabolism, Clinical and Experimental, vol. 65, no. 8, pp. 1109-1123, 2016.

[12] B. Gao and H. Tsukamoto, "Inflammation in alcoholic and nonalcoholic fatty liver disease: friend or foe?," Gastroenterology, vol. 150, no. 8, pp. 1704-1709, 2016.

[13] S. Bala, T. Csak, B. Saha et al., "The pro-inflammatory effects of miR-155 promote liver fibrosis and alcohol- induced steatohepatitis," Journal of Hepatology, vol. 64, no. 6, pp. 13781387, 2016.

[14] A. Iracheta-Vellve, C. D. Calenda, J. Petrasek et al., "FXR and TGR5 agonists ameliorate liver injury, steatosis, and inflammation after binge or prolonged alcohol feeding in mice," Hepatology Communications, vol. 2, no. 11, pp. 1379-1391, 2018.

[15] J. K. Long, W. Dai, Y. W. Zheng, and S. P. Zhao, "miR-122 promotes hepatic lipogenesis via inhibiting the LKB1/AMPK pathway by targeting Sirt1 in non-alcoholic fatty liver disease," Molecular Medicine, vol. 25, no. 1, p. 26, 2019.

[16] M. G. Zhao, X. P. Sheng, Y. P. Huang et al., “Triterpenic acids-enriched fraction from _Cyclocarya paliurus_ attenuates non- alcoholic fatty liver disease via improving oxidative stress and mitochondrial dysfunction," Biomedicine \& Pharmacotherapy, vol. 104, pp. 229-239, 2018.

[17] F. J. Yan, X. Wang, S. E. Wang et al., "C-Jun/C7ORF41/NF$\kappa \mathrm{B}$ axis mediates hepatic inflammation and lipid accumulation in NAFLD," Biochemical Journal, vol. 477, no. 3, pp. 691-708, 2020.

[18] J. Aschemann-Witzel, R. F. Gantriis, P. Fraga, and F. J. A. Perez-Cueto, "Plant-based food and protein trend from a business perspective: markets, consumers, and the challenges and opportunities in the future," Critical Reviews in Food Science and Nutrition, 2020.

[19] E. Deconinck, M. Vanhamme, J. L. Bothy, and P. Courselle, "A strategy based on fingerprinting and chemometrics for the detection of regulated plants in plant food supplements from the Belgian market: two case studies," Journal of Pharmaceutical and Biomedical Analysis, vol. 166, pp. 189-196, 2019.

[20] S. M. F. Jeurissen, E. J. M. Buurma-Rethans, M. H. Beukers, M. Jansen-van der Vliet, C. T. M. van Rossum, and R. C. Sprong, "Consumption of plant food supplements in the Netherlands," Food \& Function, vol. 9, no. 1, pp. 179-190, 2018.

[21] C. Contini, F. Boncinelli, E. Marone, G. Scozzafava, and L. Casini, "Drivers of plant-based convenience foods consumption: results of a multicomponent extension of the theory of planned behaviour," Food Quality and Preference, vol. 84, article 103931, 2020.

[22] J. Graca, M. Truninger, L. Junqueira, and L. Schmidt, "Consumption orientations may support (or hinder) transitions to more plant- based diets," Appetite, vol. 140, pp. 19-26, 2019.

[23] L. J. M. Alferink, N. S. Erler, R. J. de Knegt et al., "Adherence to a plant-based, high-fibre dietary pattern is related to regression of non-alcoholic fatty liver disease in an elderly population," European Journal of Epidemiology, vol. 35, no. 11, pp. 1069-1085, 2020.

[24] A. C. Adejumo, T. O. Ajayi, O. M. Adegbala et al., "Cannabis use is associated with reduced prevalence of progressive stages of alcoholic liver disease," Liver International, vol. 38, no. 8, pp. 1475-1486, 2018.

[25] A. Shang, R. Y. Gan, X. Y. Xu, Q. Q. Mao, P. Z. Zhang, and H. B. Li, "Effects and mechanisms of edible and medicinal plants on obesity: an updated review," Critical Reviews in Food Science and Nutrition, pp. 1-17, 2020.

[26] X. Meng, Y. Li, S. Li, R. Y. Gan, and H. B. Li, "Natural products for prevention and treatment of chemical-induced liver injuries," Comprehensive Reviews in Food Science and Food Safety, vol. 17, no. 2, pp. 472-495, 2018.

[27] S. Y. Cao, C. N. Zhao, X. Y. Xu et al., "Dietary plants, gut microbiota, and obesity: effects and mechanisms," Trends in Food Science \& Technology, vol. 92, pp. 194-204, 2019.

[28] Y. Li, S. Y. Cao, S. J. Lin, J. R. Zhang, R. Y. Gan, and H. B. Li, "Polyphenolic profile and antioxidant capacity of extracts from Gordonia axillaris fruits," Antioxidants, vol. 8, no. 6, p. 150, 2019.

[29] M. Razavi Zade, M. H. Telkabadi, F. Bahmani, B. Salehi, S. Farshbaf, and Z. Asemi, "The effects of DASH diet on weight loss and metabolic status in adults with nonalcoholic fatty liver disease: a randomized clinical trial," Liver International, vol. 36, no. 4, pp. 563-571, 2016.

[30] D. Rajdl, J. Racek, L. Trefil, P. Stehlik, J. Dobra, and V. Babuska, "Effect of folic acid, betaine, vitamin B6, and vitamin B12 on homocysteine and dimethylglycine levels in middle-aged men drinking white wine," Nutrients, vol. 8, no. 1, p. 34, 2016.

[31] S. M. Feng, T. Belwal, L. Li, J. Limwachiranon, X. Liu, and Z. Luo, "Phytosterols and their derivatives: potential healthpromoting uses against lipid metabolism and associated diseases, mechanism, and safety issues," Comprehensive Reviews in Food Science and Food Safety, vol. 19, no. 4, pp. 1243-1267, 2020. 
[32] L. Zhao, K. Wang, K. Wang, J. Zhu, and Z. Y. Hu, "Nutrient components, health benefits, and safety of litchi (Litchi chinensis Sonn.): a review," Comprehensive Reviews in Food Science and Food Safety, vol. 19, no. 4, pp. 2139-2163, 2020.

[33] S. A. Kim and S. Shin, "Fruit and vegetable consumption and non-alcoholic fatty liver disease among Korean adults: a prospective cohort study," Journal of Epidemiology and Community Health, vol. 74, no. 12, pp. 1035-1042, 2020.

[34] M. L. Xiao, G. D. Chen, F. F. Zeng et al., "Higher serum carotenoids associated with improvement of non-alcoholic fatty liver disease in adults: a prospective study," European Journal of Nutrition, vol. 58, no. 2, pp. 721-730, 2019.

[35] Y. Cao, C. Wang, J. Liu, Z. M. Liu, W. H. Ling, and Y. M. Chen, "Greater serum carotenoid levels associated with lower prevalence of nonalcoholic fatty liver disease in Chinese adults," Scientific Reports, vol. 5, no. 1, p. 12951, 2015.

[36] H. Emamat, H. Farhadnejad, H. Tangestani, A. Saneei Totmaj, H. Poustchi, and A. Hekmatdoost, "Association of allium vegetables intake and non-alcoholic fatty liver disease risk," Nutrition \& Food Science, vol. 50, no. 6, pp. 1075-1083, 2020.

[37] S. Zhang, Y. Gu, L. Wang et al., "Association between dietary raw garlic intake and newly diagnosed nonalcoholic fatty liver disease: a population-based study," European Journal of Endocrinology, vol. 181, no. 6, pp. 591-602, 2019.

[38] V. W. Setiawan, J. Porcel, P. Wei et al., "Coffee drinking and alcoholic and nonalcoholic fatty liver diseases and viral hepatitis in the multiethnic cohort," Clinical Gastroenterology and Hepatology, vol. 15, no. 8, pp. 1305-1307, 2017.

[39] H. K. Chung, J. S. Nam, M. Y. Lee et al., "The increased amount of coffee consumption lowers the incidence of fatty liver disease in Korean men," Nutrition, Metabolism, and Cardiovascular Diseases, vol. 30, no. 10, pp. 1653-1661, 2020.

[40] A. Hodge, S. Lim, E. Goh et al., "Coffee intake is associated with a lower liver stiffness in patients with non-alcoholic fatty liver disease, hepatitis C, and hepatitis B," Nutrients, vol. 9, no. 1, p. 56, 2017.

[41] I. Mikolasevic, V. Domislovic, T. Filipec Kanizaj et al., "Relationship between coffee consumption, sleep duration and smoking status with elastographic parameters of liver steatosis and fibrosis; controlled attenuation parameter and liver stiffness measurements," International Journal of Clinical Practice, vol. e13770, 2020.

[42] P. Shim, D. Choi, and Y. Park, "Association of blood fatty acid composition and dietary pattern with the risk of nonalcoholic fatty liver disease in patients who underwent cholecystectomy," Annals of Nutrition and Metabolism, vol. 70, no. 4, pp. 303-311, 2017.

[43] X. N. Liu, Y. Peng, S. H. Chen, and Q. Y. Sun, “An observational study on the association between major dietary patterns and non-alcoholic fatty liver disease in Chinese adolescents," Medicine, vol. 97, no. 17, p. e0576, 2018.

[44] Y. Xia, S. Zhang, Q. Zhang et al., "Insoluble dietary fibre intake is associated with lower prevalence of newlydiagnosed non-alcoholic fatty liver disease in Chinese men: a large population-based cross-sectional s," Nutrition and Metabolism, vol. 17, no. 1, p. 4, 2020.

[45] R. Tajima, T. Kimura, A. Enomoto et al., "No association between fruits or vegetables and non-alcoholic fatty liver disease in middle-aged men and women," Nutrition, vol. 61, pp. 119-124, 2019.
[46] Y. Xia, Z. L. Lu, M. Lu et al., "Raw orange intake is associated with higher prevalence of non-alcoholic fatty liver disease in an adult population," Nutrition, vol. 60, pp. 252-260, 2019.

[47] E. van Eekelen, J. W. J. Beulens, A. Geelen et al., "Consumption of alcoholic and sugar-sweetened beverages is associated with increased liver fat content in middle-aged men and women," Journal of Nutrition, vol. 149, no. 4, pp. 649-658, 2019.

[48] R. K. Barros, H. P. Cotrim, C. Daltro et al., "Nonalcoholic steatohepatitis in morbid obese patients: coffee consumption _vs._ disease severity," Annals of Hepatology, vol. 15, no. 3, pp. 350-355, 2016.

[49] N. Veronese, M. Notarnicola, A. M. Cisternino et al., "Coffee intake and liver steatosis: a population study in a mediterranean area," Nutrients, vol. 10, no. 1, p. 89, 2018.

[50] T. G. Simon, M. E. P. Trejo, I. Zeb et al., "Coffee consumption is not associated with prevalent subclinical cardiovascular disease (CVD) or the risk of CVD events, in nonalcoholic fatty liver disease: results from the multi-ethnic study of atherosclerosis," Metabolism, vol. 75, pp. 1-5, 2017.

[51] Y. Zhang, Z. Liu, T. Choudhury, M. C. Cornelis, and W. Liu, "Habitual coffee intake and risk for nonalcoholic fatty liver disease: a two-sample Mendelian randomization study," European Journal of Nutrition, 2020.

[52] K. Y. Xu, S. R. Liu, X. Zhao et al., "Treating hyperuricemia related non-alcoholic fatty liver disease in rats with resveratrol," Biomedicine \& Pharmacotherapy, vol. 110, pp. 844849, 2019.

[53] K. Jakubczyk, K. Skonieczna-Żydecka, J. Kałduńska, E. Stachowska, I. Gutowska, and K. Janda, "Effects of resveratrol supplementation in patients with non-alcoholic fatty liver disease-a meta-analysis," Nutrients, vol. 12, no. 8, p. $2435,2020$.

[54] T. Kessoku, K. Imajo, Y. Honda et al., "Resveratrol ameliorates fibrosis and inflammation in a mouse model of nonalcoholic steatohepatitis," Scientific Reports, vol. 6, no. 1, article 22251, 2016.

[55] S. B. Ding, J. J. Jiang, G. F. Zhang, Y. Bu, G. Zhang, and $\mathrm{X}$. Zhao, "Resveratrol and caloric restriction prevent hepatic steatosis by regulating SIRT1-autophagy pathway and alleviating endoplasmic reticulum stress in high-fat diet-fed rats," PLoS One, vol. 12, no. 8, article e0183541, 2017.

[56] G. Luo, B. Q. Huang, X. Qiu et al., "Resveratrol attenuates excessive ethanol exposure induced insulin resistance in rats via improving $\mathrm{NAD}(+) / \mathrm{NADH}$ ratio," Molecular Nutrition \& Food Research, vol. 61, no. 11, article 1700087, 2017.

[57] L. Valenti, P. Riso, A. Mazzocchi, M. Porrini, S. Fargion, and C. Agostoni, "Dietary anthocyanins as nutritional therapy for nonalcoholic fatty liver disease," Oxidative Medicine and Cellular Longevity, vol. 2013, Article ID 145421, 8 pages, 2013.

[58] Y. X. Zhu, R. J. Liu, Z. L. Shen, and G. Q. Cai, “Combination of luteolin and lycopene effectively protect against the "twohit" in NAFLD through Sirtl/AMPK signal pathway," Life Sciences, vol. 256, article 117990, 2020.

[59] X. Y. Xu, X. Meng, S. Li, R. Y. Gan, Y. Li, and H. B. Li, “Bioactivity, health benefits, and related molecular mechanisms of curcumin: current progress, challenges, and perspectives," Nutrients, vol. 10, no. 10, article 1553, 2018.

[60] N. Baziar and M. Parohan, "The effects of curcumin supplementation on body mass index, body weight, and waist circumference in patients with nonalcoholic fatty liver disease: 
a systematic review and dose-response meta-analysis of randomized controlled trials," Phytotherapy Research, vol. 34, no. 3, pp. 464-474, 2020.

[61] A. Shang, S. Y. Cao, X. Y. Xu et al., "Bioactive compounds and biological functions of garlic (Allium sativum L.)," Food, vol. 8, no. 7, p. 246, 2019.

[62] S. Panyod, W. K. Wu, K. H. Lu et al., "Allicin modifies the composition and function of the gut microbiota in alcoholic hepatic steatosis mice," Journal of Agricultural and Food Chemistry, vol. 68, no. 10, pp. 3088-3098, 2020.

[63] H. M. Xia, J. Wang, X. J. Xie, L. J. Xu, and S. Q. Tang, “Green tea polyphenols attenuate hepatic steatosis, and reduce insulin resistance and inflammation in high-fat diet-induced rats," International Journal of Molecular Medicine, vol. 44, no. 4, pp. 1523-1530, 2019.

[64] L. T. Ferreira, C. P. B. de Sousa Filho, M. P. Marinovic, A. C. Rodrigues, and R. Otton, "Green tea polyphenols positively impact hepatic metabolism of adiponectin- knockout lean mice," Journal of Functional Foods, vol. 64, article 103679, 2020.

[65] Z. Yang, M. Z. Zhu, Y. B. Zhang et al., "Coadministration of epigallocatechin-3-gallate (EGCG) and caffeine in low dose ameliorates obesity and nonalcoholic fatty liver disease in obese rats," Phytotherapy Research, vol. 33, no. 4, pp. 10191026, 2019.

[66] G. Y. Tang, C. N. Zhao, X. Y. Xu et al., "Phytochemical composition and antioxidant capacity of 30 Chinese teas," Antioxidants, vol. 8, no. 6, p. 180, 2019.

[67] W. Y. Khoo, B. J. Chrisfield, S. Sae-Tan, and J. D. Lambert, "Mitigation of nonalcoholic fatty liver disease in high-fatfed mice by the combination of decaffeinated green tea extract and voluntary exercise," Journal of Nutritional Biochemistry, vol. 76, article 108262, 2020.

[68] F. Wang, Y. Li, Y. J. Zhang, Y. Zhou, S. Li, and H. B. Li, "Natural products for the prevention and treatment of hangover and alcohol use disorder," Molecules, vol. 21, no. 1, p. 64, 2016.

[69] O. J. Kennedy, P. Roderick, R. Poole, and J. Parkes, "Coffee, caffeine and non-alcoholic fatty liver disease?," Therapeutic Advances in Gastroenterology, vol. 9, no. 3, pp. 417-418, 2016.

[70] P. Shokouh, P. B. Jeppesen, K. Hermansen et al., "A combination of coffee compounds shows insulin-sensitizing and hepatoprotective effects in a rat model of diet-induced metabolic syndrome," Nutrients, vol. 10, no. 1, p. 6, 2018.

[71] M. Bagherniya, V. Nobili, C. N. Blesso, and A. Sahebkar, "Medicinal plants and bioactive natural compounds in the treatment of non- alcoholic fatty liver disease: A clinical review," Pharmacological Research, vol. 130, pp. 213-240, 2018.

[72] L. Abenavoli, A. A. Izzo, N. Milić, C. Cicala, A. Santini, and R. Capasso, "Milk thistle (Silybum marianum): a concise overview on its chemistry, pharmacological, and nutraceutical uses in liver diseases," Phytotherapy Research, vol. 32, no. 11, pp. 2202-2213, 2018.

[73] Z. R. Yang, H. F. Wang, T. C. Zuo, L. L. Guan, and N. Dai, "Salidroside alleviates oxidative stress in the liver with nonalcoholic steatohepatitis in rats," BMC Pharmacology and Toxicology, vol. 17, no. 1, p. 16, 2016.

[74] T. Zheng, X. Y. Yang, W. J. Li et al., "Salidroside attenuates high-fat diet-induced nonalcoholic fatty liver disease via AMPK-dependent TXNIP/NLRP3 pathway," Oxidative
Medicine and Cellular Longevity, vol. 2018, Article ID 8597897, 17 pages, 2018.

[75] C. Colica, L. Boccuto, and L. Abenavoli, "Silymarin: an option to treat non-alcoholic fatty liver disease," World Journal of Gastroenterology, vol. 23, no. 47, pp. 8437-8438, 2017.

[76] E. Sahin, R. Bagci, N. E. Bektur Aykanat, S. Kacar, and V. Sahinturk, "Silymarin attenuated nonalcoholic fatty liver disease through the regulation of endoplasmic reticulum stress proteins GRP78 and XBP-1 in mice," Journal of Food Biochemistry, vol. 44, no. 6, article e13194, 2020.

[77] H. J. Won, H. I. Kim, T. Park et al., "Non-clinical pharmacokinetic behavior of ginsenosides," Journal of Ginseng Research, vol. 43, no. 3, pp. 354-360, 2019.

[78] Y. Yao, "Ginsenosides reduce body weight and ameliorate hepatic steatosis in high fat diet-induced obese mice via endoplasmic reticulum stress and p-STAT3/STAT3 signaling," Molecular Medicine Reports, vol. 21, no. 3, pp. 1059-1070, 2020.

[79] S. Wan, L. Zhang, Y. Quan, and K. Wei, "Resveratrol-loaded PLGA nanoparticles: enhanced stability, solubility and bioactivity of resveratrol for non-alcoholic fatty liver disease therapy," Royal Society Open Science, vol. 5, no. 11, article 181457, 2018.

[80] Y. Huang, H. Lang, K. Chen et al., "Resveratrol protects against nonalcoholic fatty liver disease by improving lipid metabolism and redox homeostasis via the PPAR $\alpha$ pathway," Applied Physiology, Nutrition, and Metabolism, vol. 45, no. 3, pp. 227-239, 2020.

[81] H. Hosseini, M. Teimouri, M. Shabani et al., "Resveratrol alleviates non-alcoholic fatty liver disease through epigenetic modification of the Nrf2 signaling pathway," International Journal of Biochemistry \& Cell Biology, vol. 119, article 105667, 2020.

[82] Q. Liu, R. Pan, L. Ding et al., "Rutin exhibits hepatoprotective effects in a mouse model of non-alcoholic fatty liver disease by reducing hepatic lipid levels and mitigating lipid- induced oxidative injuries," International Immunopharmacology, vol. 49, pp. 132-141, 2017.

[83] Q. Chu, S. Zhang, M. Chen et al., "Cherry anthocyanins regulate NAFLD by promoting autophagy pathway," Oxidative Medicine and Cellular Longevity, vol. 2019, Article ID 4825949, 16 pages, 2019.

[84] M. Fan, Y. J. Choi, Y. Tang, S. M. Bae, H. P. Yang, and E. K. Kim, "Efficacy and mechanism of polymerized anthocyanin from grape-skin extract on high-fat-diet-induced nonalcoholic fatty liver disease," Nutrients, vol. 11, no. 11, article 2586, 2019.

[85] F. J. García-Alonso, R. González-Barrio, G. Martín-Pozuelo et al., "A study of the prebiotic-like effects of tomato juice consumption in rats with diet-induced non-alcoholic fatty liver disease (NAFLD)," Food \& Function, vol. 8, no. 10, pp. 3542-3552, 2017.

[86] M. Róvero Costa, J. Leite Garcia, C. Cristina Vágula de Almeida Silva et al., "Lycopene modulates pathophysiological processes of non-alcoholic fatty liver disease in obese rats," Antioxidants, vol. 8, no. 8, p. 276, 2019.

[87] R. M. Piña-Zentella, J. L. Rosado, M. A. Gallegos-Corona, L. A. Madrigal-Pérez, O. P. García, and M. Ramos-Gomez, "Lycopene improves diet-mediated recuperation in rat model of nonalcoholic fatty liver disease," Journal of Medicinal Food, vol. 19, no. 6, pp. 607-614, 2016. 
[88] W. Jiang, M. H. Guo, and X. Hai, "Hepatoprotective and antioxidant effects of lycopene on non-alcoholic fatty liver disease in rat," World Journal of Gastroenterology, vol. 22, no. 46, pp. 10180-10188, 2016.

[89] Y. Hu, F. Yin, Z. Liu et al., "Acerola polysaccharides ameliorate high-fat diet-induced non-alcoholic fatty liver disease through reduction of lipogenesis and improvement of mitochondrial functions in mice," Food \& Function, vol. 11, no. 1, pp. 1037-1048, 2020.

[90] D. K. Yang and D. G. Jo, "Mulberry fruit extract ameliorates nonalcoholic fatty liver disease (NAFLD) through inhibition of mitochondrial oxidative stress in rats," Evidence-based Complementary and Alternative Medicine, vol. 2018, Article ID 8165716, 9 pages, 2018.

[91] M. M. F. Carvalho, L. L. T. Reis, J. M. M. Lopes et al., “Açai improves non-alcoholic fatty liver disease (NAFLD) induced by fructose," Nutrición Hospitalaria, vol. 35, no. 2, pp. 318$325,2018$.

[92] L. Y. Tang, Y. Chen, B. B. Rui, and C. M. Hu, "Resveratrol ameliorates lipid accumulation in HepG2 cells, associated with down-regulation of lipin1 expression," Canadian Journal of Physiology and Pharmacology, vol. 94, no. 2, pp. 185189, 2016.

[93] J. Gao, S. Chen, Z. Qiu et al., "Myricitrin ameliorates ethanolinduced steatosis in mouse AML12 liver cells by activating AMPK, and reducing oxidative stress and expression of inflammatory cytokines," Molecular Medicine Reports, vol. 17, no. 5, pp. 7381-7387, 2018.

[94] C. Guo, G. Xue, B. Pan et al., "Myricetin ameliorates ethanolinduced lipid accumulation in liver cells by reducing fatty acid biosynthesis," Molecular Nutrition \& Food Research, vol. 63, no. 14, article 1801393, 2019.

[95] T. Zhou, Y. J. Zhang, D. P. Xu et al., "Protective effects of lemon juice on alcohol-induced liver injury in mice," BioMed Research International, vol. 2017, Article ID 7463571, 8 pages, 2017.

[96] M. Cheng, J. Zhu, T. Ren, and M. Zhou, "The combination of blueberry juice and probiotics reduces apoptosis of alcoholic fatty liver of mice by affecting SIRT1 pathway," Drug Design, Development and Therapy, vol. 10, pp. 1649-1661, 2016.

[97] Q. Zhuge, Y. Zhang, B. Liu, and M. Wu, "Blueberry polyphenols play a preventive effect on alcoholic fatty liver disease C57BL/6 J mice by promoting autophagy to accelerate lipolysis to eliminate excessive TG accumulation in hepatocytes," Annals of Palliative Medicine, vol. 9, no. 3, pp. 1045-1054, 2020.

[98] J. Xiao, R. Zhang, F. Huang et al., "The biphasic dose effect of lychee (Litchi chinensis Sonn.) pulp phenolic extract on alcoholic liver disease in mice," Food \& Function, vol. 8, no. 1, pp. 189-200, 2017.

[99] J. Xiao, R. Zhang, F. Huang et al., "Lychee (Litchi chinensis Sonn.) pulp phenolic extract confers a protective activity against alcoholic liver disease in mice by alleviating mitochondrial dysfunction," Journal of Agricultural and Food Chemistry, vol. 65, no. 24, pp. 5000-5009, 2017.

[100] S. Park, D. S. Kim, X. Wu, and Q. J Yi, "Mulberry and dandelion water extracts prevent alcohol-induced steatosis with alleviating gut microbiome dysbiosis," Experimental Biology and Medicine, vol. 243, no. 11, pp. 882-894, 2018.

[101] J. Zhou, J. Zhang, C. Wang et al., “Açaí (Euterpe oleracea Mart.) attenuates alcohol-induced liver injury in rats by alleviating oxidative stress and inflammatory response," Experi- mental and Therapeutic Medicine, vol. 15, no. 1, pp. 166172,2018

[102] C. Yan, Y. Zhang, X. Zhang, J. Aa, G. Wang, and Y. Xie, “Curcumin regulates endogenous and exogenous metabolism via Nrf2-FXR-LXR pathway in NAFLD mice," Biomedicine \& Pharmacotherapy, vol. 105, pp. 274-281, 2018.

[103] M. E. Inzaugarat, E. de Matteo, P. Baz et al., "New evidence for the therapeutic potential of curcumin to treat nonalcoholic fatty liver disease in humans," PLoS One, vol. 12, no. 3, article e0172900, 2017.

[104] D. E. Lee, S. J. Lee, S. J. Kim, H. S. Lee, and O. S. Kwon, "Curcumin ameliorates nonalcoholic fatty liver disease through inhibition of O-GlcNAcylation," Nutrients, vol. 11, no. 11, article 2702, 2019.

[105] H. Emamat, F. Foroughi, H. Eini-Zinab, M. Taghizadeh, M. Rismanchi, and A. Hekmatdoost, "The effects of onion consumption on treatment of metabolic, histologic, and inflammatory features of nonalcoholic fatty liver disease," Journal of Diabetes \& Metabolic Disorders, vol. 15, no. 1, p. 25, 2015.

[106] H. Emamat, F. Foroughi, H. Eini-Zinab, and A. Hekmatdoost, "The effects of onion consumption on prevention of nonalcoholic fatty liver disease," Indian Journal of Clinical Biochemistry, vol. 33, no. 1, pp. 75-80, 2018.

[107] S. Panyod, W. K. Wu, C. T. Ho et al., "Diet supplementation with allicin protects against alcoholic fatty liver disease in mice by improving anti-inflammation and antioxidative functions," Journal of Agricultural and Food Chemistry, vol. 64, no. 38, pp. 7104-7113, 2016.

[108] A. Shirpoor, E. Heshmati, F. Kheradmand et al., "Increased hepatic FAT/CD36, PTP1B and decreased HNF4A expression contributes to dyslipidemia associated with ethanolinduced liver dysfunction: rescue effect of ginger extract," Biomedicine \& Pharmacotherapy, vol. 105, pp. 144-150, 2018.

[109] C. Guo, J. Ma, Q. Zhong et al., "Curcumin improves alcoholic fatty liver by inhibiting fatty acid biosynthesis," Toxicology and Applied Pharmacology, vol. 328, pp. 1-9, 2017.

[110] L. F. Torres, B. Cogliati, and R. Otton, "Green tea prevents NAFLD by modulation of miR-34a and miR-194 expression in a high-fat diet mouse model," Oxidative Medicine and Cellular Longevity, vol. 2019, Article ID 4168380, 18 pages, 2019.

[111] B. Liu, J. Zhang, P. Sun, R. Yi, X. Han, and X. Zhao, "Raw bowl tea (Tuocha) polyphenol prevention of nonalcoholic fatty liver disease by regulating intestinal function in mice," Biomolecules, vol. 9, no. 9, p. 435, 2019.

[112] L. Braud, S. Battault, G. Meyer et al., "Antioxidant properties of tea blunt ROS-dependent lipogenesis: beneficial effect on hepatic steatosis in a high fat-high sucrose diet NAFLD obese rat model," Journal of Nutritional Biochemistry, vol. 40, pp. 95-104, 2017.

[113] A. Labdi, M. Amiali, Y. N. Bachir et al., "Green tea extract attenuates non alcoholic fatty liver disease by decreasing hyperlipidemia and enhancing superoxide dismutase activity in cholesterol-fed rats," Mediterranean Journal of Nutrition and Metabolism, vol. 11, no. 3, pp. 295-306, 2018.

[114] Y. Tan, J. Kim, J. Cheng et al., "Green tea polyphenols ameliorate non-alcoholic fatty liver disease through upregulating AMPK activation in high fat fed Zucker fatty rats," World Journal of Gastroenterology, vol. 23, no. 21, pp. 3805-3814, 2017. 
[115] Y. Shen, X. Xiao, K. Wu et al., "Effects and molecular mechanisms of Ninghong black tea extract in nonalcoholic fatty liver disease of rats," Journal of Food Science, vol. 85, no. 3, pp. 800-807, 2020.

[116] F. Wang, Y. J. Zhang, Y. Zhou et al., "Effects of beverages on alcohol metabolism: potential health benefits and harmful impacts," International Journal of Molecular Sciences, vol. 17, no. 3, p. 354, 2016.

[117] K. H. Chen, P. C. Li, W. H. Lin, C. T. Chien, and B. H. Low, "Depression by a green tea extract of alcohol-induced oxidative stress and lipogenesis in rat liver," Bioscience Biotechnology and Biochemistry, vol. 75, no. 9, pp. 1668-1676, 2014.

[118] G. E. Arteel, T. Uesugi, L. N. Bevan et al., "Green tea extract protects against early alcohol-induced liver injury in rats," Biological Chemistry, vol. 383, no. 3-4, pp. 663-670, 2002.

[119] J. W. Yun, Y. K. Kim, B. S. Lee et al., "Effect of dietary epigallocatechin-3-gallate on cytochrome P450 2E1dependent alcoholic liver damage: enhancement of fatty acid oxidation," Bioscience Biotechnology and Biochemistry, vol. 71, no. 12, pp. 2999-3006, 2014.

[120] S. Bharrhan, A. Koul, K. Chopra, and P. Rishi, "Catechin suppresses an array of signalling molecules and modulates alcohol-induced endotoxin mediated liver injury in a rat model," PLoS One, vol. 6, no. 6, article e20635, 2011.

[121] H. M. Kim, Y. Kim, E. S. Lee, J. H. Huh, and C. H. Chung, "Caffeic acid ameliorates hepatic steatosis and reduces ER stress in high fat diet-induced obese mice by regulating autophagy," Nutrition, vol. 55-56, pp. 63-70, 2018.

[122] L. Sharma, N. A. Lone, R. M. Knott, A. Hassan, and T. Abdullah, "Trigonelline prevents high cholesterol and high fat diet induced hepatic lipid accumulation and lipo-toxicity in C57BL/6J mice, via restoration of hepatic autophagy," Food and Chemical Toxicology, vol. 121, pp. 283-296, 2018.

[123] S. J. Zhang, Y. F. Li, G. E. Wang et al., "Caffeine ameliorates high energy diet-induced hepatic steatosis: sirtuin 3 acts as a bridge in the lipid metabolism pathway," Food \& Function, vol. 6, no. 8, pp. 2578-2587, 2015.

[124] S. Watanabe, T. Takahashi, H. Ogawa et al., "Daily coffee intake inhibits pancreatic beta cell damage and nonalcoholic steatohepatitis in a mouse model of spontaneous metabolic syndrome, tsumura-suzuki obese diabetic mice," Metabolic Syndrome and Related Disorders, vol. 15, no. 4, pp. 170177, 2017.

[125] A. Ontawong, O. Boonphang, T. Pasachan et al., "Hepatoprotective effect of coffee pulp aqueous extract combined with simvastatin against hepatic steatosis in high-fat dietinduced obese rats," Journal of Functional Foods, vol. 54, pp. 568-577, 2019.

[126] P. Vitaglione, G. Mazzone, V. Lembo et al., "Coffee prevents fatty liver disease induced by a high-fat diet by modulating pathways of the gut-liver axis," Journal of Nutritional Science, vol. 8, p. e15, 2019.

[127] D. F. Zhang, F. Zhang, J. Zhang, R. M. Zhang, and R. Li, "Protection effect of trigonelline on liver of rats with nonalcoholic fatty liver diseases," Asian Pacific Journal of Tropical Medicine, vol. 8, no. 8, pp. 639-642, 2015.

[128] C. Y. Fang, X. B. Cai, S. Hayashi et al., "Caffeine-stimulated muscle IL-6 mediates alleviation of non-alcoholic fatty liver disease," Biochimica et Biophysica Acta-Molecular and Cell Biology of Lipids, vol. 1864, no. 3, pp. 271-280, 2019.
[129] A. M. Velázquez, N. Roglans, R. Bentanachs et al., "Effects of a low dose of caffeine alone or as part of a green coffee extract, in a rat dietary model of lean non-alcoholic fatty liver disease without inflammation," Nutrients, vol. 12, no. 11, article 3240, 2020.

[130] P. Shokouh, P. B. Jeppesen, K. Hermansen et al., "Effects of unfiltered coffee and bioactive coffee compounds on the development of metabolic syndrome components in a highfat-/high-fructose-fed rat model," Nutrients, vol. 10, no. 10, article 1547, 2018.

[131] A. Brandt, A. Nier, C. J. Jin et al., "Consumption of decaffeinated coffee protects against the development of early nonalcoholic steatohepatitis: role of intestinal barrier function," Redox Biology, vol. 21, article 101092, 2019.

[132] S. W. Hu, L. P. Xia, H. W. Luo et al., "Prenatal caffeine exposure increases the susceptibility to non-alcoholic fatty liver disease in female offspring rats _via_ activation of GRC/EBP $\alpha$-SIRT1 pathway," Toxicology, vol. 417, pp. 23-34, 2019.

[133] X. W. Lv, Z. Chen, J. Li et al., "Caffeine protects against alcoholic liver injury by attenuating inflammatory response and oxidative stress," Inflammation Research, vol. 59, no. 8, pp. 635-645, 2010.

[134] Q. Wang, X. F. Dai, W. Z. Yang et al., "Caffeine protects against alcohol-induced liver fibrosis by dampening the cAMP/PKA/CREB pathway in rat hepatic stellate cells," International Immunopharmacology, vol. 25, no. 2, pp. 340352, 2015.

[135] K. Karthikesan and L. Pari, "Caffeic acid alleviates the increased lipid levels of serum and tissues in alcoholinduced rats," Fundamental \& Clinical Pharmacology, vol. 22, no. 5, pp. 523-527, 2008.

[136] L. Lin, Z. Hao, S. Zhang et al., "Study on the protection of water extracts of Polygoni multiflori radix and Polygoni multiflori radix praeparata against NAFLD and its mechanism," Journal of Ethnopharmacology, vol. 252, article 112577, 2020.

[137] Q. Feng, C. Liu, W. Gao, X. L. Geng, and N. Dai, "Salidrosidemitigated inflammatory injury of hepatocytes with nonalcoholic fatty liver disease via inhibition TRPM2 ion channel activation," Diabetes Metabolic Syndrome and ObesityTargets and Therapy, vol. 12, pp. 2755-2763, 2019.

[138] G. Vecchione, E. Grasselli, F. Cioffi et al., "The nutraceutic silybin counteracts excess lipid accumulation and ongoing oxidative stress in an in vitro model of non-alcoholic fatty liver disease progression," Frontiers in Nutrition, vol. 4, p. $42,2017$.

[139] R. Sun, D. Xu, Q. Wei et al., "Silybin ameliorates hepatic lipid accumulation and modulates global metabolism in an NAFLD mouse model," Biomedicine \& Pharmacotherapy, vol. 123, article 109721, 2020

[140] H. S. Li, H. Ying, and Z. Y. He, "Salidroside and curcumin formula prevents liver injury in nonalcoholic fatty liver disease in rats," Annals of Hepatology, vol. 17, no. 5, pp. 769$778,2018$.

[141] Y. Meng, Y. Liu, N. Fang, and Y. Guo, "Hepatoprotective effects of Cassia semen ethanol extract on non-alcoholic fatty liver disease in experimental rat," Pharmaceutical Biology, vol. 57, no. 1, pp. 98-104, 2019.

[142] Q. Wu, H. Tang, and H. Wang, "The anti-oxidation and mechanism of essential oil of Paederia scandens in the NAFLD model of chicken," Animals, vol. 9, no. 10, p. 850, 2019. 
[143] L. Yu, L. Gong, C. Wang et al., "Radix polygoni multiflori and its main component emodin attenuate non-alcoholic fatty liver disease in zebrafish by regulation of AMPK signaling pathway," Drug Design, Development and Therapy, vol. 14, pp. 1493-1506, 2020.

[144] C. F. Hu, L. P. Sun, Q. H. Yang, D. X. Lu, and S. Luo, "Ginsenosides from stems and leaves of ginseng prevent ethanolinduced lipid accumulation in human L02 hepatocytes," Chinese Journal of Integrative Medicine, vol. 23, no. 6, pp. 438444, 2017.

[145] R. Y. Choi, M. J. Woo, J. R. Ham, and M. K. Lee, “Anti-steatotic and anti-inflammatory effects of _Hovenia dulcis_ Thunb. extracts in chronic alcohol-fed rats," Biomedicine \& Pharmacotherapy, vol. 90, pp. 393-401, 2017.

[146] J. T. Wu, G. W. Yang, C. H. Qi, L. Zhou, J. G. Hu, and M. S. Wang, "ANTI-INFLAMMATORYACTIVITY of platycodin $\mathrm{D}$ on alcohol-induced fatty liver rats via TLR4-MYD88NF-," African Journal of Traditional, Complementary, and Alternative Medicines, vol. 13, no. 4, pp. 176-183, 2016.

[147] C. Y. Bang, J. H. Byun, H. K. Choi, J. S. Choi, and S. Y. Choung, "Protective effects of Ecklonia stolonifera extract on ethanol-induced fatty liver in rats," Biomolecules \& Therapeutics, vol. 24, no. 6, pp. 650-658, 2016.

[148] Q. Q. Mao, X. Y. Xu, S. Y. Cao et al., "Bioactive compounds and bioactivities of ginger (Zingiber officinale Roscoe)," Food, vol. 8, no. 6, p. 185, 2019.

[149] S. Li, L. Q. Gan, S. K. Li, J. C. Zheng, D. P. Xu, and H. B. Li, "Effects of herbal infusions, tea and carbonated beverages on alcohol dehydrogenase and aldehyde dehydrogenase activity," Food \& Function, vol. 5, no. 1, pp. 42-49, 2014.

[150] S. Y. Cao, B. Y. Li, R. Y. Gan et al., "The in vivo antioxidant and hepatoprotective actions of selected Chinese teas," Food, vol. 9, no. 3, p. 262, 2020.

[151] C. N. Zhao, G. Y. Tang, S. Y. Cao et al., "Phenolic profiles and antioxidant activities of 30 tea infusions from green, black, oolong, white, yellow and dark teas," Antioxidants, vol. 8, no. 7, p. 215, 2019.

[152] A. Calabrò, A. C. Procopio, F. Primerano et al., "Beneficial effects of coffee in non-alcoholic fatty liver disease: a narrative review," Hepatoma Research, vol. 2020, p. 69, 2020.

[153] M. Rezaei-Tavirani, M. R. Tavirani, M. Z. Azodi, Z. Akbari, and H. Hajimehdipoor, "Prediction of coffee effects in rats with healthy and NAFLD conditions based on proteinprotein interaction network analysis," Research Journal of Pharmacognosy, vol. 6, no. 4, pp. 7-15, 2019.

[154] Q. Liu, X. Meng, Y. Li et al., "Natural products for the prevention and management of Helicobacter pylori infection," Comprehensive Reviews in Food Science and Food Safety, vol. 17, no. 4, pp. 937-952, 2018.

[155] D. P. Xu, Y. Li, X. Meng et al., "Natural antioxidants in foods and medicinal plants: extraction, assessment and resources," International Journal of Molecular Sciences, vol. 18, no. 1, p. 96, 2017.

[156] R. Negri, G. Trinchese, F. Carbone et al., "Randomised clinical trial: calorie restriction regimen with tomato juice supplementation ameliorates oxidative stress and preserves a proper immune surveillance modulating mitochondrial bioenergetics of T-lymphocytes in obese children affected by nonalcoholic fatty liver disease (NAFLD)," Journal of Clinical Medicine, vol. 9, no. 1, p. 141, 2020.
[157] A. C. Kaliora, A. Kokkinos, A. Diolintzi et al., "The effect of minimal dietary changes with raisins in NAFLD patients with non-significant fibrosis: a randomized controlled intervention," Food \& Function, vol. 7, no. 11, pp. 45334544, 2016.

[158] M. Krawczyk, D. Maciejewska, K. Ryterska et al., "Gut permeability might be improved by dietary fiber in individuals with nonalcoholic fatty liver disease (NAFLD) undergoing weight reduction," Nutrients, vol. 10, no. 11, p. 1793, 2018.

[159] M. Daneshi-Maskooni, S. A. Keshavarz, M. Qorbani et al., "Green cardamom increases sirtuin-1 and reduces inflammation in overweight or obese patients with non-alcoholic fatty liver disease: a double-blind randomized placebo-controlled clinical trial," Nutrition and Metabolism, vol. 15, no. 1, p. 63, 2018.

[160] S. Rahmani, S. Asgary, G. Askari et al., "Treatment of nonalcoholic fatty liver disease with curcumin: a randomized placebo-controlled trial," Phytotherapy Research, vol. 30, no. 9, pp. 1540-1548, 2016.

[161] M. Saberi-Karimian, M. Keshvari, M. Ghayour-Mobarhan et al., "Effects of curcuminoids on inflammatory status in patients with non-alcoholic fatty liver disease: a randomized controlled trial," Complementary Therapies in Medicine, vol. 49, p. 102322, 2020.

[162] S. Chashmniam, S. R. Mirhafez, M. Dehabeh, M. Hariri, M. Azimi Nezhad, and B. F. Nobsakht M Gh, "A pilot study of the effect of phospholipid curcumin on serum metabolomic profile in patients with non-alcoholic fatty liver disease: a randomized, double-blind, placebo-controlled trial," European Journal of Clinical Nutrition, vol. 73, no. 9, pp. 1224$1235,2019$.

[163] N. Rostampour, K. A. Kasiri, E. Hashemi-Dehkordi, A. M. Taheri, A. F. Broujeni, and M. Rafieian-Kopaei, “Therapeutic effects of green tea on nonalcoholic fatty liver disease in 1016-year-old children," Journal of Clinical and Diagnostic Research, vol. 13, no. 7, pp. Bc4-Bc7, 2019.

[164] M. Hussain, R. Habib Ur, and L. Akhtar, "Therapeutic benefits of green tea extract on various parameters in nonalcoholic fatty liver disease patients," Pakistan Journal of Medical Sciences, vol. 33, no. 4, pp. 931-936, 2017.

[165] M. Mahmoodi, R. Hosseini, A. Kazemi, R. Ofori-Asenso, M. Mazidi, and S. M. Mazloomi, "Effects of green tea or green tea catechin on liver enzymes in healthy individuals and people with nonalcoholic fatty liver disease: a systematic review and meta-analysis of randomized clinical trials," Phytotherapy Research, vol. 34, no. 7, pp. 1587-1598, 2020.

[166] S. Hosseinabadi, M. Rafraf, A. Mahmoodzadeh, M. AsghariJafarabadi, and S. Asghari, "Effects of green coffee extract supplementation on glycemic indexes, leptin, and obesity values in patients with non-alcoholic fatty liver disease," Journal of Herbal Medicine, vol. 22, article 100340, 2020.

[167] S. Hosseinabadi, M. Rafraf, S. Asghari, M. Asghari-Jafarabadi, and S. Vojouhi, "Effect of green coffee extract supplementation on serum adiponectin concentration and lipid profile in patients with non-alcoholic fatty liver disease: a randomized, controlled trial," Complementary Therapies in Medicine, vol. 49, article 102290, 2020.

[168] A. Mansour, M. R. Mohajeri-Tehrani, S. Karimi et al., "Short term effects of coffee components consumption on gut microbiota in patients with non-alcoholic fatty liver and diabetes: a pilot randomized placebo-controlled, clinical trial," EXCLI Journal, vol. 19, pp. 241-250, 2020. 
[169] H. Shen, A. C. Rodriguez, A. Shiani et al., "Association between caffeine consumption and nonalcoholic fatty liver disease: a systemic review and meta-analysis," Therapeutic Advances in Gastroenterology, vol. 9, no. 1, pp. 113-120, 2015.

[170] M. Dorosti, A. Jafary Heidarloo, F. Bakhshimoghaddam, and M. Alizadeh, "Whole-grain consumption and its effects on hepatic steatosis and liver enzymes in patients with nonalcoholic fatty liver disease: a randomised controlled clinical trial," British Journal of Nutrition, vol. 123, no. 3, pp. 328336, 2020.

[171] M. Dinu, A. Whittaker, G. Pagliai et al., “A Khorasan wheatbased replacement diet improves risk profile of patients with nonalcoholic fatty liver disease (NAFLD): a randomized clinical trial," Journal of the American College of Nutrition, vol. 37, no. 6, pp. 508-514, 2018.

[172] S. M. R. Hosseini, G. R. G. Razmgah, M. Nematy et al., "Efficacy of black seed (Nigella sativa) and lemon balm (Melissa officinalis) on non-alcoholic fatty liver disease: a randomized controlled clinical trial," Iranian Red Crescent Medical Journal, vol. 20, no. 3, article e59183, 2018.

[173] A. Khonche, H. F. Huseini, M. Gholamian, R. Mohtashami, F. Nabati, and S. Kianbakht, "Standardized_Nigella sativa_ seed oil ameliorates hepatic steatosis, aminotransferase and lipid levels in non-alcoholic fatty liver disease: A randomized, double-blind and placebo-controlled clinical trial," Journal of Ethnopharmacology, vol. 234, pp. 106-111, 2019.

[174] M. Darand, Z. Darabi, Z. Yari et al., “_Nigella sativa_ and inflammatory biomarkers in patients with non-alcoholic fatty liver disease: Results from a randomized, double-blind, placebo- controlled, clinical trial," Complementary Therapies in Medicine, vol. 44, pp. 204-209, 2019.

[175] N. Khavasi, M. H. Somi, E. Khadem et al., "Effect of daily caper fruit pickle consumption on disease regression in patients with non-alcoholic fatty liver disease: a doubleblinded randomized clinical trial," Advanced Pharmaceutical Bulletin, vol. 7, no. 4, pp. 645-650, 2017.

[176] N. Khavasi, M. Somi, E. Khadem, M. H. Ayati, M. Torbati, and S. M. Fazljou, "Daily consumption of the capparis spinosa reduces some atherogenic indices in patients with non-alcoholic fatty liver disease: a randomized, double-blind, clinical trial," Iranian Red Crescent Medical Journal, vol. 20, no. 11, article e63446, 2018.

[177] Z. Yari, M. Cheraghpour, S. M. Alavian, M. Hedayati, H. Eini-Zinab, and A. Hekmatdoost, "The efficacy of flaxseed and hesperidin on non-alcoholic fatty liver disease: an openlabeled randomized controlled trial," European Journal of Clinical Nutrition, vol. 75, no. 1, pp. 99-111, 2021.

[178] C. Sergides, M. Chirila, L. Silvestro, D. Pitta, and A. Pittas, "Bioavailability and safety study of resveratrol $500 \mathrm{mg}$ tablets in healthy male and female volunteers," Experimental and Therapeutic Medicine, vol. 11, no. 1, pp. 164-170, 2016.

[179] S. D. Anton, C. Embry, M. Marsiske et al., "Safety and metabolic outcomes of resveratrol supplementation in older adults: results of a twelve-week, placebo-controlled pilot study," Experimental Gerontology, vol. 57, pp. 181-187, 2014.

[180] V. S. Chachay, G. A. Macdonald, J. H. Martin et al., "Resveratrol does not benefit patients with nonalcoholic fatty liver disease," Clinical Gastroenterology and Hepatology, vol. 12, no. 12, pp. 2092-2103.e6, 2014.

[181] H. Y. Liu, T. B. Walden, D. Cai et al., "Dietary fiber in bilberry ameliorates pre-obesity events in rats by regulating lipid depot, cecal short-chain fatty acid formation and microbiota composition," Nutrients, vol. 11, no. 6, article 1350, 2019.

[182] D. Bagchi, S. Roy, V. Patel et al., "Safety and whole-body antioxidant potential of a novel anthocyanin-rich formulation of edible berries," Molecular and Cellular Biochemistry, vol. 281, no. 1-2, pp. 197-209, 2006.

[183] A. J. Vargas, L. N. McDonnell, Z. F. Liu, B. C. Wertheim, C. A. Thomson, and P. A. Thompson, "A pilot sweet cherry feeding study in overweight men: tolerance, safety, and anthocyanin exposure," Journal of Functional Foods, vol. 11, pp. 500-508, 2014.

[184] F. Salomone, J. Godos, and S. Zelber-Sagi, "Natural antioxidants for non-alcoholic fatty liver disease: molecular targets and clinical perspectives," Liver International, vol. 36, no. 1, pp. 5-20, 2016.

[185] P. W. Zhang, F. X. Chen, D. Li, W. H. Ling, and H. H. Guo, "A CONSORT-compliant, randomized, double-blind, placebo-controlled pilot trial of purified anthocyanin in patients with nonalcoholic fatty liver disease," Medicine, vol. 94, no. 20, article e758, 2015.

[186] M. Honda, T. Higashiura, and T. Fukaya, "Safety assessment of a natural tomato oleoresin containing high amounts of Zisomers of lycopene prepared with supercritical carbon dioxide," Journal of the Science of Food and Agriculture, vol. 97, no. 3, pp. 1027-1033, 2017.

[187] A. Nier, A. Brandt, I. B. Conzelmann, Y. Özel, and I. Bergheim, "Non-alcoholic fatty liver disease in overweight children: role of fructose intake and dietary pattern," Nutrients, vol. 10, no. 9, article 1329, 2018.

[188] M. Rokutan, D. Yabe, I. Komoto et al., “A case of insulinoma with non-alcoholic fatty liver disease: roles of hyperphagia and hyperinsulinemia in pathogenesis of the disease," Endocrine Journal, vol. 62, no. 11, pp. 1025-1030, 2015.

[189] Y. Panahi, P. Kianpour, R. Mohtashami, R. Jafari, L. E. Simental-Mendía, and A. Sahebkar, "Efficacy and safety of phytosomal curcumin in non-alcoholic fatty liver disease: a randomized controlled trial," Drug Research, vol. 67, no. 4, pp. 244-251, 2017.

[190] Y. Panahi, P. Kianpour, R. Mohtashami, S. S. Soflaei, and A. Sahebkar, "Efficacy of phospholipidated curcumin in nonalcoholic fatty liver disease: a clinical study," Journal of Asian Natural Products Research, vol. 21, no. 8, pp. 798-805, 2019.

[191] D. Soleimani, Z. Paknahad, and M. H. Rouhani, “Therapeutic effects of garlic on hepatic steatosis in nonalcoholic fatty liver disease patients: a randomized clinical Trial," Diabetes Metabolic Syndrome and Obesity-Targets and Therapy, vol. Volume 13, pp. 2389-2397, 2020.

[192] B. Salehi, P. Zucca, I. E. Orhan et al., "Allicin and health: a comprehensive review," Trends in Food Science \& Technology, vol. 86, pp. 502-516, 2019.

[193] S. Kaviarasan, P. Viswanathan, M. K. Ravichandran, and C. V. Anuradha, "(-) Epigallocatechin gallate (EGCG) prevents lipid changes and collagen abnormalities in chronic ethanol-fed rats," Toxicology Mechanisms and Methods, vol. 18, no. 5, pp. 425-432, 2008.

[194] W. Y. Hu, X. H. Ma, W. Y. Zhou, X. X. Li, T. T. Sun, and H. Sun, "Preventive effect of silibinin in combination with pu-erh tea extract on non-alcoholic fatty liver disease in ob/ob mice," Food \& Function, vol. 8, no. 3, pp. 1105-1115, 2017. 
[195] S. M. Tabatabaee, S. M. Alavian, L. Ghalichi et al., "Green tea in non-alcoholic fatty liver disease: a double blind randomized clinical trial," Hepatitis Monthly, vol. 17, no. 12, article e14993, 2017.

[196] A. Pezeshki, S. Safi, A. Feizi, G. Askari, and F. Karami, “The effect of green tea extract supplementation on liver enzymes in patients with nonalcoholic fatty liver disease," International Journal of Preventive Medicine, vol. 7, p. 131, 2016.

[197] H. H. S. Chow, Y. Cai, I. A. Hakim et al., "Pharmacokinetics and safety of green tea polyphenols after multiple-dose administration of epigallocatechin gallate and polyphenon $\mathrm{E}$ in healthy individuals," Clinical Cancer Research, vol. 9, no. 9, pp. 3312-3319, 2003.

[198] W. C. S. Faria, A. A. da Silva, N. Veggi et al., "Acute and subacute oral toxicity assessment of dry encapsulated and nonencapsulated green coffee fruit extracts," Journal of Food and Drug Analysis, vol. 28, no. 2, pp. 143-161, 2020.

[199] K. L. Kendall, J. R. Moon, C. M. Fairman et al., "Ingesting a preworkout supplement containing caffeine, creatine, $\beta_{-}$ -alanine, amino acids, and B vitamins for 28 days is both safe and efficacious in recreationally active men," Nutrition Research, vol. 34, no. 5, pp. 442-449, 2014.

[200] N. A. de Oliveira, T. M. Sandini, H. P. Cornelio-Santiago et al., "Acute and subacute ( 28 days) toxicity of green coffee oil enriched with diterpenes cafestol and kahweol in rats," Regulatory Toxicology and Pharmacology, vol. 110, article 104517, 2020.

[201] A. Iriondo-DeHond, F. S. Cornejo, B. Fernandez-Gomez et al., "Bioaccesibility, metabolism, and excretion of lipids composing spent coffee grounds," Nutrients, vol. 11, no. 6, article 1411, 2019.

[202] V. J. Navarro, S. H. Belle, M. D'Amato et al., "Silymarin in non-cirrhotics with non-alcoholic steatohepatitis: a randomized, double-blind, placebo controlled trial," PLoS One, vol. 14, no. 9, article e0221683, 2019.

[203] J. B. Zhu, X. Y. Wan, Y. P. Zhu, X. Ma, Y. Zheng, and T. Zhang, "Evaluation of salidroside in vitro and in vivo genotoxicity," Drug and Chemical Toxicology, vol. 33, no. 2, pp. 220-226, 2010.

[204] B. Wicklow, K. Wittmeier, G. W. t' Jong et al., "Proposed trial: safety and efficacy of resveratrol for the treatment of nonalcoholic fatty liver disease (NAFLD) and associated insulin resistance in adolescents who are overweight or obese adolescents - rationale and protocol," Biochemistry and Cell Biology, vol. 93, no. 5, pp. 522-530, 2015.

[205] Z. S. Sangsefidi, M. Hosseinzadeh, A. M. Ranjbar, M. Akhondi-Meybodi, H. Fallahzadeh, and H. MozaffariKhosravi, "The effect of total anthocyanin-base standardized (Cornus mas L.) fruit extract on liver function, tumor necrosis factor $\alpha$, malondealdehyde, and adiponectin in patients with non-alcoholic fatty liver: a study protocol for a doubleblind randomized clinical trial," Nutrition Journal, vol. 18, no. 1, p. 39, 2019. 